OPEN ACCESS

Edited by:

Robert J. Harvey,

University of the Sunshine Coast,

Australia

Reviewed by:

Joe Lynch,

The University of Queensland,

Australia

Julia Dallman,

University of Miami, United States

${ }^{*}$ Correspondence:

Hiromi Hirata

hihirata@chem.aoyama.ac.jp

${ }^{\dagger}$ Deceased 9 May 2018

Received: 26 April 2018

Accepted: 30 July 2018

Published: 03 September 2018

Citation:

Low SE, Ito $D$ and Hirata H

(2018) Characterization of the

Zebrafish Glycine Receptor Family

Reveals Insights Into Glycine

Receptor Structure Function and

Stoichiometry.

Front. Mol. Neurosci. 11:286. doi: 10.3389/fnmol.2018.00286

\section{Characterization of the Zebrafish Glycine Receptor Family Reveals Insights Into Glycine Receptor Structure Function and Stoichiometry}

\author{
Sean Eric Low ${ }^{\dagger}$, Daishi Ito and Hiromi Hirata* \\ Department of Chemistry and Biological Science, Aoyama Gakuin University, Sagamihara, Japan
}

To study characterization of zebrafish glycine receptors (zGlyRs), we assessed expression and function of five $\alpha$ - and two B-subunit encoding GlyR in zebrafish. Our qPCR analysis revealed variable expression during development, while in situ hybridizations uncovered expression in the hindbrain and spinal cord; a finding consistent with the reported expression of GlyR subunits in these tissues from other organisms. Electrophysiological recordings using Xenopus oocytes revealed that all five $\alpha$ subunits form homomeric receptors activated by glycine, and inhibited by strychnine and picrotoxin. In contrast, B subunits only formed functional heteromeric receptors when co-expressed with $\alpha$ subunits. Curiously, the second transmembranes of both $B$ subunits were found to lack a phenylalanine at the sixth position that is commonly associated with conferring picrotoxin resistance to heteromeric receptors. Consistent with the absence of phenylalanines at the sixth position, heteromeric zGlyRs often lacked significant picrotoxin resistance. Subsequent efforts revealed that resistance to picrotoxin in both zebrafish and human heteromeric GlyRs involves known residues within transmembrane 2 , as well as previously unknown residues within transmembrane 3. We also found that a dominant mutation in human GlyR $\alpha 1$ that gives rise to hyperekplexia, and recessive mutations in zebrafish GlyRßb that underlie the bandoneon family of motor mutants, result in reduced receptor function. Lastly, through the use of a concatenated construct we demonstrate that zebrafish heteromeric receptors assemble with a stoichiometry of $3 \alpha: 2 \beta$. Collectively, our findings have furthered our knowledge regarding the assembly of heteromeric receptors, and the molecular basis of $B$ subunit-conferred picrotoxin resistance. These results should aid in future investigations of glycinergic signaling in zebrafish and mammals.

Keywords: zebrafish, glycine receptor, picrotoxin, sensorimotor, hyperekplexia

\section{INTRODUCTION}

Glycine, the major inhibitory neurotransmitter in the brain stem and spinal cord, contributes to the control of motor pattern generation, the synchronization of spinal reflexes, and the processing of sensory stimuli (Lynch, 2004, 2009; Betz and Laube, 2006; Dutertre et al., 2012). Glycine exerts its effect through the opening of chloride-permeable channels termed glycine receptors (GlyRs). Structurally, GlyRs belong to the Cys-loop superfamily of receptors that in vertebrates includes 
chloride-permeable GABA receptors, and the cationic-permeable acetylcholine, serotonin and glutamate receptors. As a member of this family, GlyRs are comprised of five subunits, each possessing a large $\mathrm{N}$-terminal extracellular domain and four transmembrane domains, the second of which lines the pore (Pribilla et al., 1992; Bormann et al., 1993; Du et al., 2015; Gielen et al., 2015; Huang et al., 2015). In addition to comprising the inter-subunit binding site for glycine, and the competitive antagonist strychnine, the N-terminal domains of all GlyR subunits contain a signal peptide sequence that promotes the translocation of mRNA-bound ribosomes to the ER whereupon translation continues. Thereafter, the signal peptide is cleaved and subunits oligomerize into pentameric receptors comprised of either all $\alpha$ subunits, or a mix of $\alpha$ and $\beta$ subunits, before being allowed to traffic to the plasma membrane. Although $B$ subunits lack the ability to form functional homomeric receptors, due to an absence of critical residues within several N-terminal assembly motifs (Griffon et al., 1999), ß subunits are nonetheless essential for the synaptic localization of GlyRs owing to the ability of the intracellular loop between transmembranes three and four of $B$ subunits ( $B$-loop) to bind to the postsynaptic scaffolding gephyrin (Meyer et al., 1995). This requirement of B subunits for the synaptic localization of GlyRs has raised questions regarding the subunit stoichiometry of heteromeric GlyRs, with conflicting experimental evidence in support of either $3 \alpha: 2 \beta$ or $2 \alpha: 3 \beta$ (Langosch et al., 1988; Kuhse et al., 1993; Burzomato et al., 2003; Grudzinska et al., 2005; Durisic et al., 2012; Yang et al., 2012).

To date four members of the zebrafish family have been characterized in detail. The first, zebrafish GlyR (zGlyR) $\alpha 1$ was found to form functional homomeric receptors when expressed in Xenopus oocytes and mammalian cells (David-Watine et al., 1999). The second and third zGlyR subunits identified were tentatively named zGlyR $\alpha 2$ and zGlyRß (Imboden et al., 2001a,c), however, subsequent phylogenetic analysis and completion of the zebrafish genome necessitated their reassignments as zGlyR $\alpha 4 a$ and zGlyRßa, respectively (Imboden et al., 2001b). Curiously, the initially recovered signal peptide sequence of $z G l y R \alpha 4 a$ was found to be insufficient for the generation of glycineevoked currents, while a chimera containing the signal peptide sequence from $z G l y R \alpha 1$ resulted in functional homomeric zGlyR $\alpha 4 a$ receptors (Imboden et al., 2001a). Although this finding raised the possibility that zebrafish zGlyR $\alpha 4 a$ might represent a pseudogene similar to human GlyR $\alpha 4$ (Simon et al., 2004; Leacock et al., 2018), a subsequent study found that an alternate variant of zGlyR $\alpha 4$ a containing a different signal peptide sequence was capable of compensating for the loss of GlyR expression in zebrafish (Hirata et al., 2013), a finding consistent with the alternate variant being functional. Lastly, several zebrafish mutants uncovered in forward genetic screens for abnormal sensory-evoked motor behaviors have been found to arise from mutations in zGlyRßb (Granato et al., 1996; Hirata et al., 2005; Ganser et al., 2013). Mutations in zGlyRßb, collectively known as bandoneon mutants, result in bilateral muscle contractions in the trunk and tail due to a loss of reciprocal inhibition in the spinal cord. Similarly, mutations in human GlyRß and GlyR $\alpha 1$ have been shown to cause an excessive startle response disorder known as hyperekplexia (Shiang et al., 1993; Rees et al., 2002; Chung et al., 2013; James et al., 2013). This finding demonstrates the utility of using zebrafish to gain insight into human neurological disorders, and highlights the need for a better understanding of the zGlyR family.

In order to gain a more complete picture of zGlyRs we cloned and characterized each subunit. These efforts revealed that the family is comprised of five $\alpha$ and two $\beta$ subunits that exhibit variable expression patterns during development. All five $\alpha$ subunits form homomeric receptors, while $\beta$ subunits complex with $\alpha$ subunits at a stoichiometry of $3 \alpha: 2 \beta$ to form heteromeric receptors. Subsequent analysis of mutations linked to motor impairment in zebrafish uncovered that mutated subunits often formed hypomorphic receptors. Lastly, zGlyRß subunits typically failed to confer significant picrotoxin resistance to heteromeric $\mathrm{zGlyR} \alpha / \beta$ receptors in accordance with the composition of amino acids in transmembrane domain 2. A closer inspection found that known residues in transmembrane domain 2, as well as previously unknown residues in transmembrane domain 3 of GlyRß subunits contribute to picrotoxin resistance in both zebrafish and human heteromeric receptors.

\section{MATERIALS AND METHODS}

\section{Reagents}

Unless otherwise noted, all chemicals and reagents were obtained from Wako Pure Chemical Industries and Thermo Fisher Scientific, and used according to manufacturer's guidelines.

\section{Animal Care and Use}

Zebrafish were bred and used according to protocols set forth by the institutional animal care and use committee at Aoyama Gakuin University. Embryos reared in a $28.5^{\circ} \mathrm{C}$ incubator were staged using established guidelines (Kimmel et al., 1995), and are given as hours post-fertilization (hpf).

\section{Molecular Biology, qPCR and in situ Hybridization}

Full-length cDNAs encoding zGlyR subunits were obtained by RT-PCR using an oligo-dT primer, SuperScript ${ }^{\circledR}$ IV, and total RNA harvested from $48 \mathrm{hpf}$ to $60 \mathrm{hpf}$ larvae with TRIzol ${ }^{\circledR}$. To each cDNA in pCS2+ a Kozak sequence of GCCGCCACC was added before the initial methionine codon to promote translation. Concatemers were constructed by substitution of zGlyR $\alpha 1$ 's stop codon with non-redundant nucleotides encoding a 7-fold repeat of alanine-glycine-serine, followed by the post-signal peptide sequence of zGlyRßb. Site-directed mutagenesis was performed using $50 \mathrm{ng}$ of donor template, $20 \mathrm{pmol}$ of mutagenic primers, and 1 unit of Phusion ${ }^{\circledR}$ High-Fidelity DNA Polymerase (New England Biolabs) in a final volume of $50 \mu \mathrm{l}$. Prior to transformation the donor template was destroyed by adding 10 units of DpnI and incubating at $37^{\circ} \mathrm{C}$ for $1-2 \mathrm{~h}$. Capped cRNAs for expression in Xenopus laevis oocytes were synthesized from 
linearized templates using an SP6 mMessage mMachine ${ }^{\circledR}$ kit (Ambion).

qPCR analysis was performed on cDNA synthesized from total RNA extracted from 50 to 100 embryos/larvae for each stage using gene-specific primers (Table 1) and KAPA FAST SYBR ${ }^{\mathrm{TM}}$ according to manufacturer's guidelines. Relative expression levels were quantified using: $2 \wedge(C t(\beta$ actin $)-C t(z G l y R$ subunit)).

Templates for zGlyR riboprobes were isolated using primers raised against either divergent coding sequence or 3' untranslated regions (Table 1). DIG-conjugated riboprobes were synthesized and used according to established procedures (Low et al., 2011). Images were captured using a ProgRes ${ }^{\circledR}$ CF Scan camera and associated software (Jenoptik), where after auto contrast in Photoshop CS3 (Adobe) was employed.

\section{Electrophysiology}

Oocytes were injected with five femtomoles of cRNA using a Nanoject II (Drummond Scientific), where after oocytes were incubated in Barth's solution (in mM: $88 \mathrm{NaCl}, 1 \mathrm{KCl}$, $2.4 \mathrm{NaHCO}_{3}, 0.33 \mathrm{Ca}\left(\mathrm{NO}_{3}\right)_{2}, 0.41 \mathrm{CaCl}_{2}, 0.82 \mathrm{MgSO}_{4}, 10 \mathrm{HEPES}$ at $\mathrm{pH} 7.5$ with $\mathrm{NaOH}$, supplemented with gentamicin at $50 \mu \mathrm{g} / \mathrm{ml}$, and penicillin/streptomycin at $100 \mathrm{units} / \mathrm{ml}$ ) at $17^{\circ} \mathrm{C}$ for $24-72 \mathrm{~h}$ prior to recording. To determine the distribution of heteromeric receptor assembly we used the following equation: Probability of receptor subtype $=(K)^{*} p^{\alpha *}(1-p)^{\beta}$, where " $p$ " is the proportion of cRNA encoding a subunit, " $\alpha$ " and " $\beta$ " are the numbers of $\alpha$ and $\beta$ subunits in a receptor subtype, and " $K$ " is the number of ways a receptor subtype can form during receptor assembly: 1 for $5 \alpha: 0 \beta$ and $0 \alpha: 5 \beta$, 5 for $4 \alpha: 1 \beta$ and $1 \alpha: 4 \beta$, and 10 for $3 \alpha: 2 \beta$ and $2 \alpha: 3 \beta$. Please note that functional homomeric $\beta$ receptors $(0 \alpha: 5 ß)$ are not made when determining the percentage of receptor subtypes. Oocyte recording solution (in $\mathrm{mM}: 90 \mathrm{NaCl}, 1 \mathrm{KCl}, 2 \mathrm{CaCl}_{2}$, $1 \mathrm{MgCl}_{2}, 10$ HEPES at $\mathrm{pH} 7.5$ with $\mathrm{NaOH}$ ) and up to seven other solutions were applied to oocytes using a BPS-8 solution switcher (ALA Scientific). Borosilicate electrodes had resistances of $\sim 0.5 \mathrm{M} \Omega$ when filed with $3 \mathrm{M} \mathrm{KCl}$. Two-electrode voltage- clamp recordings were made from oocytes held at $-50 \mathrm{mV}$ using pClamp $^{\mathrm{TM}} 10.2$ to control a GeneClamp ${ }^{\circledR} 500 \mathrm{~B}$ amplifier via a Digidata ${ }^{\circledR} 1440$ A digitizer (Molecular Devices). Signals were low-pass filtered at $10 \mathrm{~Hz}$, and sampled at $100 \mathrm{~Hz}$. Recordings were analyzed using Clampfit 10.7 (Axon Instruments) and SigmaPlot 11.0 (Systat Software, Inc.). Statistical significance was assessed using a Mann-Whitney $U$ test assuming equal variance at $p<0.05$.

\section{Imaging}

HEK293T cells $\left(5 \times 10^{5}\right)$ were transfected with $2 \mu \mathrm{g}$ of expression vectors using the Lipofectamine2000 method according to the manufactures' protocol. Frame averaged $(6 \times)$ optical slices $(x / y$; 4096/4096) of live HEK293T cells were captured at $400 \mathrm{~Hz}$ using a $40 \times$ objective (HCX APO L, NA 0.8, water) and a TCS SP5 laserscanning confocal microscope (Leica) with a pinhole diameter of 1 Airy. Gain settings that were below saturation for venus-tagged zGlyR $\alpha 1$ transfected cells were subsequently used to acquire fluorescent images of venus-tagged $z G l y R \alpha 1^{\mathrm{R} 271 \mathrm{Q}}$ transfected cells. Images were prepared using Fiji (Schindelin et al., 2012) and Adobe Photoshop CS3 wherein the Blur filter was used.

\section{RESULTS}

\section{Zebrafish Glycine Receptor Subunits Share Structural Similarities With Human Subunits}

An assessment of the GRCz11 genome assembly indicated the presence of seven putative open reading frames encoding zGlyR subunits (Figure 1A). Subsequent cloning and sequence analysis revealed that zebrafish possess single orthologs of $\alpha 1, \alpha 2$ and $\alpha 3$, and two orthologs of $\alpha 4$ ( $\alpha 4 \mathrm{a}$ and $\alpha 4 \mathrm{~b})$ and $\beta$ ( $\beta \mathrm{a}$ and $\beta b)$. Phylogenetic analysis revealed that $\alpha 3$ subfamily is close to $\alpha 1$ subfamily, and $\alpha 4$ close to $\alpha 2$ (Figure 1B). The duplicated $\alpha 4$ and $\beta$ subunits are herein referred to as paralogs in accordance with established guidelines (Wood, 1998).

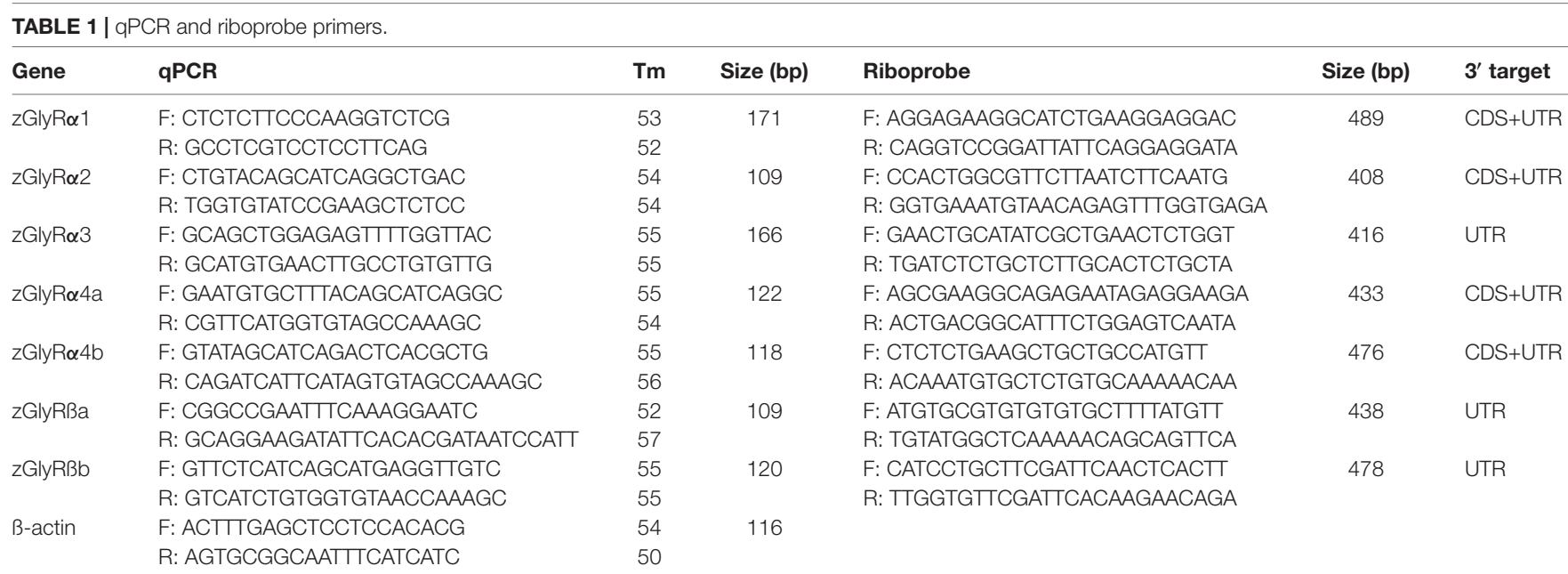

(F) forward and (R) reverse primer, (Tm) melting temperature, (CDS) coding sequence, (UTR) untranslated region. 
A

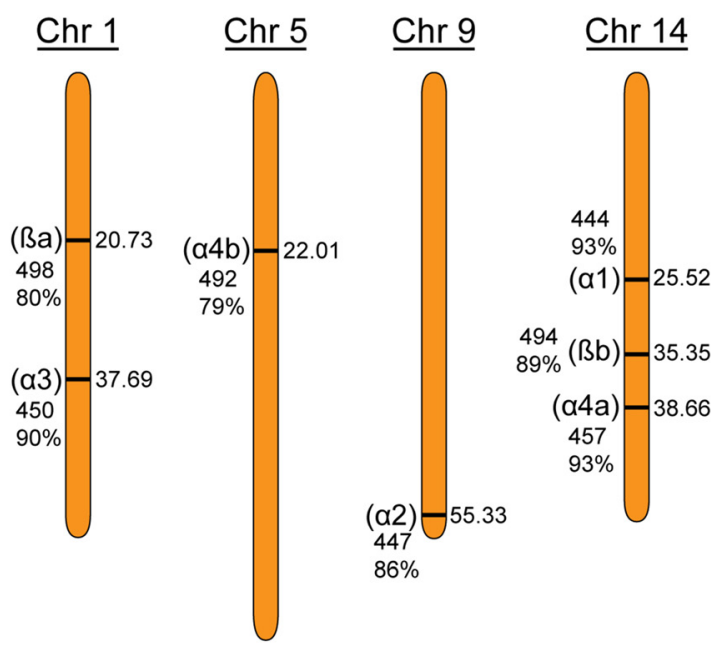

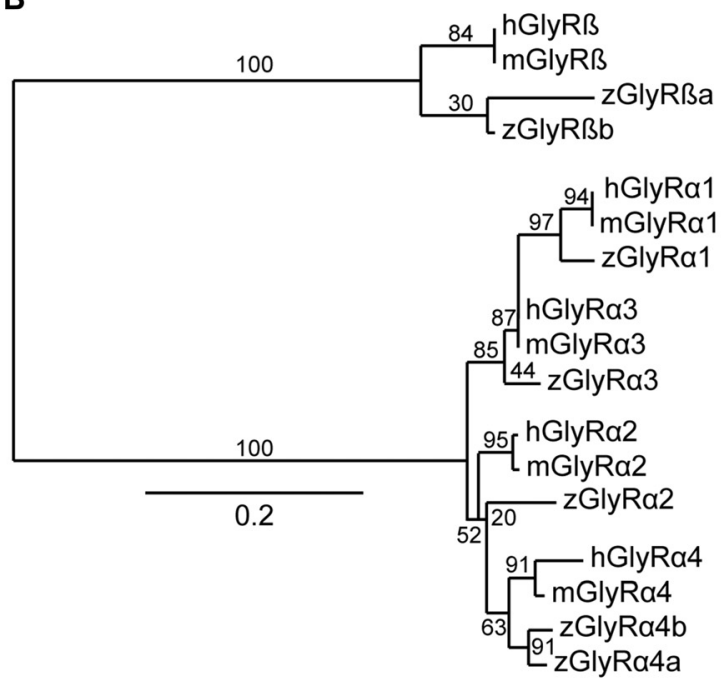

C

$\mathrm{zGlyR} \alpha 1$ $z G l y R \alpha 2$ zGlyR $\alpha 3$ zGlyR $\alpha 4 a$ $z G l y R \alpha 4 b$ hGlyR $\alpha 1$

zGlyRßa zGlyRßb $h G l_{Y R} \beta$

$$
\begin{array}{ll}
\text { AD1 } \\
35 & \text { PPVN. .63 } \\
39 & \text { PPVN. .67 } \\
40 & \text { PPVN. .68 } \\
42 & \text { PPVN. . 70 } \\
40 & \text { PPVN. .68 } \\
35 & \text { PPVN. .63 } \\
55 & \text { IPVE. .83 } \\
56 & \text { IPVE. .84 } \\
56 & \text { IPVD. .84 }
\end{array}
$$$$
(-) B D
$$$$
(+) B D
$$$$
\sum<
$$$$
<
$$

$(+) \mathrm{BD}$

M2

FLR. . 157 ESF. . 200

FLR..161 ESF..204

FLR. 162 ESF..205

FLR. 164 ESF. . 207

FLR. 162

ESF. . 205

FLR. . 157

ESF. . 200

FLR. . 178 ESF..221

FLR. . 179 ESF..222

FLR. .180 ESF..223

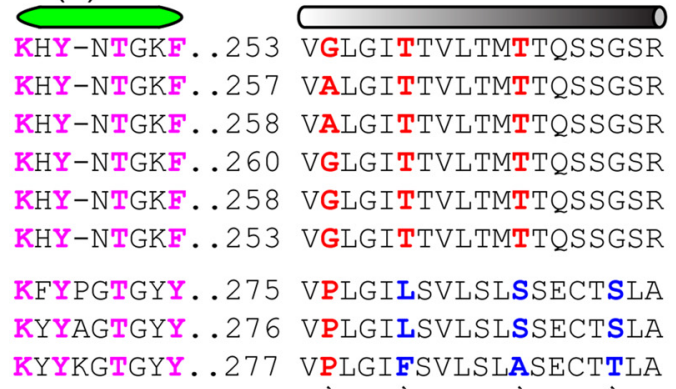

$i$ i 6 i

FIGURE 1 | Zebrafish family of glycine receptors (GlyR). (A) Chromosomal (Chr) arrangement of zebrafish GlyR (zGlyR) subunits. Location (in Mbp) are on the right, while name of subunit, length and sequence similarity to human ortholog are given to the left. (B) Phylogenetic analysis of zGlyR subunits. Horizontal scale bar at the bottom reflects amount of genetic change, all other numbers are bootstraps. (mGlyR and hGlyR) mouse and human GlyR subunit. (C) Sequence alignments of zGlyR $\alpha$ subunits to hGlyR $\alpha 1$, and zGlyRB subunits to hGlyRB. Red letters indicate residues conserved among $\alpha$ and $B$ subunits from zebrafish and human. Blue letters indicate residues conserved between zGlyRß paralogs which differ from hGlyRß. Magenta letters indicate residues conserved between $\alpha$ and $B$ subunits from zebrafish and human. AD1: assembly domain one, (-)BD and (+)BD: negative and positive faces of the inter-subunit binding sites for glycine and strychnine, M2: transmembrane domain 2. Numbered residues (") indicate position with M2.

A closer inspection of the amino acid content of each zGlyR subunit revealed the following notable features. Residues thought to form the positive $(+)$ and negative $(-)$ portions of the inter-subunit binding sites for glycine and strychnine (Vandenberg et al., 1992; Grudzinska et al., 2005), as well as the receptor's preference for glycine over closely related molecules such as B-alanine, GABA and D-serine (Schmieden et al., 1993), are conserved (Figure 1C). In addition, the proline and asparagine residues in assembly domain one that are essential for oligomerization of GlyR subunits in the ER, a necessary step in the translocation of assembled GlyRs out of the ER (Griffon et al., 1999), are present in all five zGlyR $\alpha$ subunits, but are absent in both zGlyRß subunits. Therefore, like their mammalian orthologs, zGlyRß subunits are unlikely to form functional homomeric receptors.

In a previous study, the second transmembrane domains (M2s) of GlyR subunits have been shown to be related to function (Shan et al., 2001). The second residue (2') of the M2 within GlyR $\alpha$ subunits is occupied by a glycine or alanine, as opposed to a proline typical of $\beta$ subunits (Figure 1C). While a glycine or alanine at the 2' position in the human GlyR $\alpha 1$ subunit ( $\mathrm{hGlyR} \alpha 1^{\mathrm{G} 254 \mathrm{~A}}$ ) was found to have no effect on the excitatory amount of glycine required to half-maximally activate receptors $\left(\mathrm{EC}_{50}\right)$, substitution to

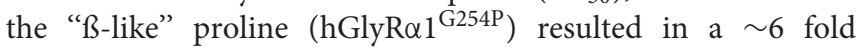
increase in the $\mathrm{EC}_{50}$ for glycine (Shan et al., 2001). The other intriguing feature of M2 was the presence of a leucine at the 6' position in zGlyRß subunits, which is normally occupied by a phenylalanine in $B$ subunits from the other vertebrate species (Hirata et al., 2009). The phenylalanine at the 6 ' position has been shown to be both necessary for $\beta$ subunit-conferred picrotoxin resistance to heteromeric hGlyR $\alpha 1 / \beta$ receptors, and sufficient to confer resistance to homomeric hGlyR $\alpha 1$ receptors (Shan et al., 2001). Taken 


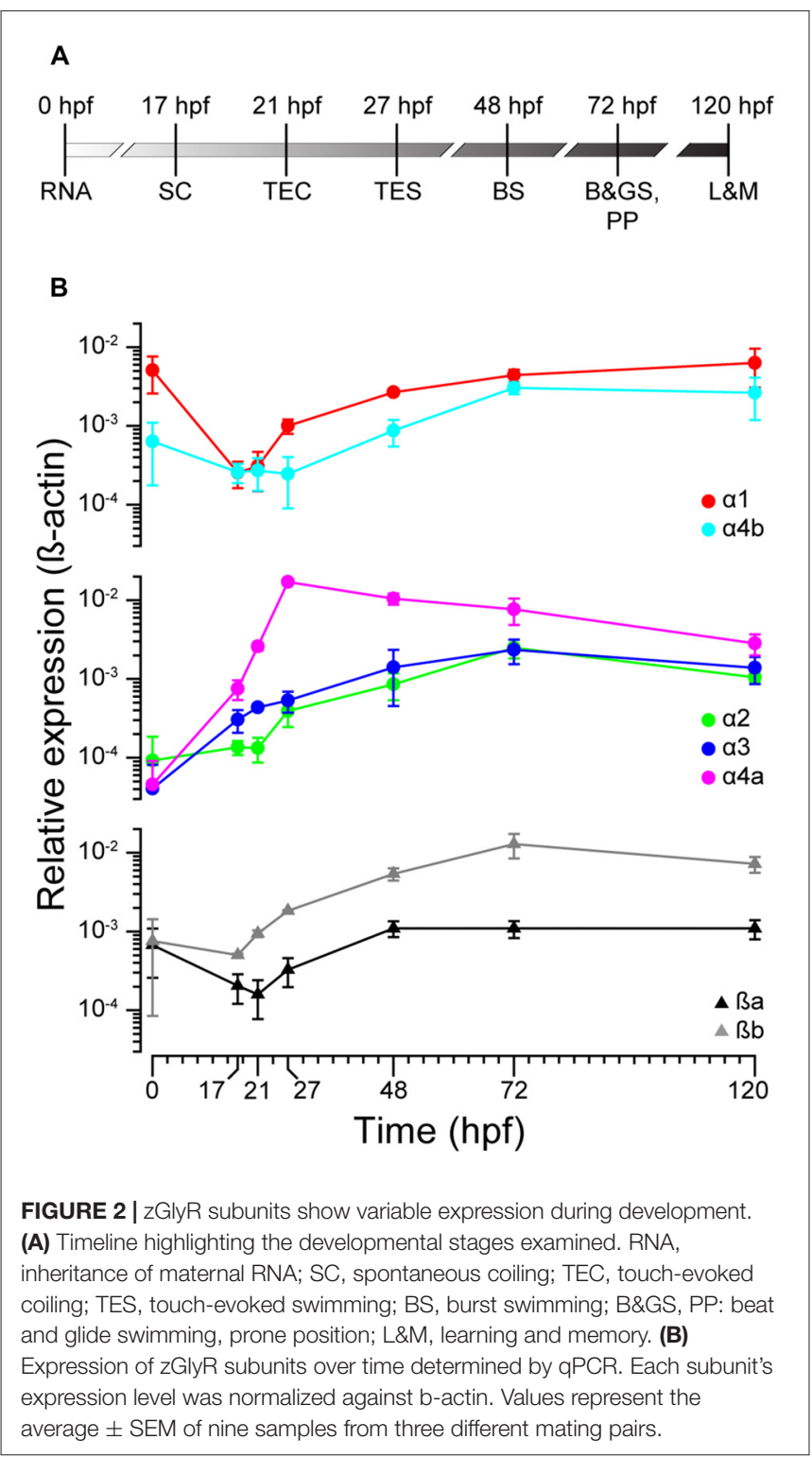

together, homomeric $\mathrm{zGlyR} \alpha$ receptors would be expected to exhibit moderate $\mathrm{EC}_{50 s}$ for glycine, while heteromeric zGlyR $\alpha 1 / \beta$ receptors might manifest as receptors with reduced picrotoxin resistance.

\section{zGlyR Subunits Exhibit Variable Expression in the Nervous System During Development}

To ascertain the expression profiles of zGlyR subunits during development, we performed qPCR at time points that coincided with the following (Figure 2A): the inheritance of maternal RNA $(0 \mathrm{hpf})$, the onset of spontaneous motor activity (17 hpf), and the emergence of touch-evoked contractions ( $21 \mathrm{hpf}$ ) and swimming (27 hpf), both of which involve glycine-dependent reciprocal inhibition between the bilateral halves of the spinal cord (SaintAmant and Drapeau, 2000; Hirata et al., 2005). In addition, we examined commonly employed stages of development including

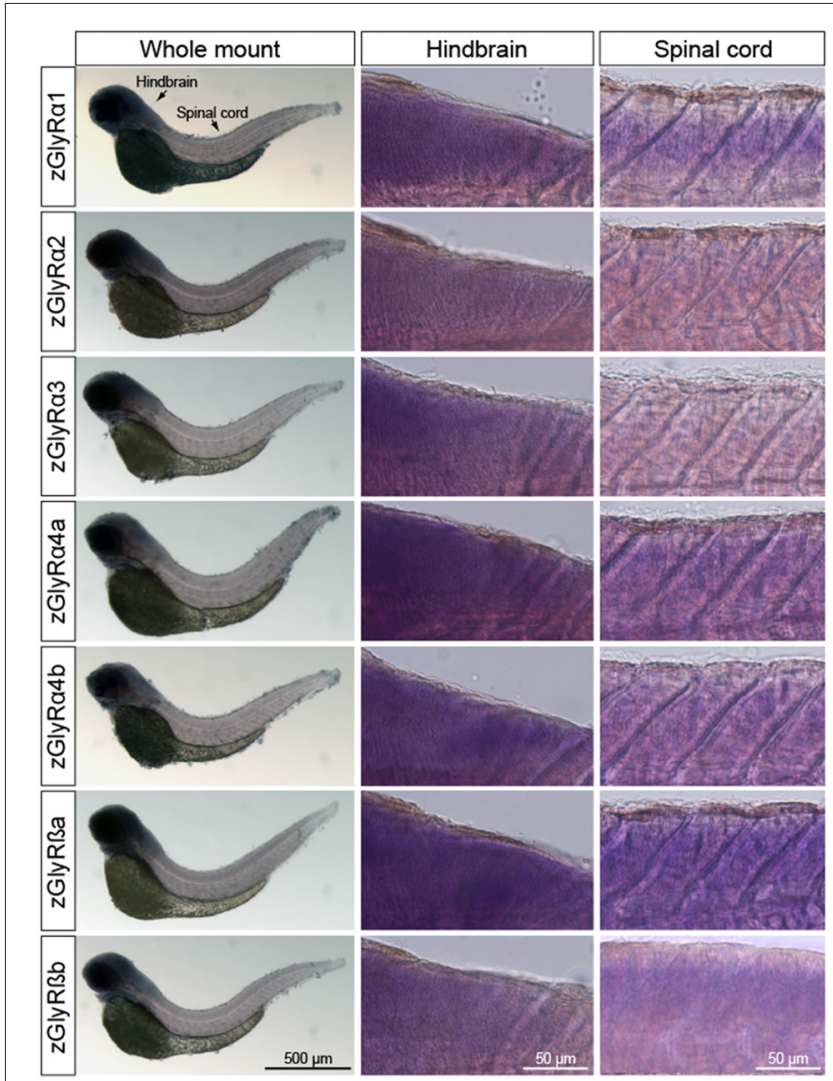

FIGURE 3 | Whole-mount in situ hybridizations of zGlyR subunits from 48 hours post-fertilization (hpf) larvae. Individual subunits are indicated to the left.

when embryos exhibit "burst" swimming (48 hpf), larvae adopt the prone position and convert to "beat and glide" swimming (72 hpf), and when larvae possess many adult-like abilities such as learning and memory (120 hpf). We found that transcripts encoding $z G l y R \alpha 1$ and $z G l y R \alpha 4 b$ were dominant among the zGlyR $\alpha$ subunits in inherited maternal RNA (Figure 2B), while RNA encoding zGlyRßa and zGlyRßb subunits were equally represented. Thereafter, RNA encoding these four subunits all displayed a modest reduction at the onset of spontaneous motor activity, while at the same time RNA encoding zGlyR $\alpha 2$ remained constant, and RNA encoding zGlyR $\alpha 3$ and zGlyR $\alpha 4 a$ increased. The cumulative effect resulted in a decrease in the extent of RNA variation from 125 -fold at $0 \mathrm{hpf}$ (zGlyR $\alpha 1$ vs. zGlyR $\alpha 3$ ) to 6 -fold at $17 \mathrm{hpf}(\mathrm{zGlyR} \alpha 4 \mathrm{a}$ vs. zGlyR $\alpha 2)$. Thereafter, all RNAs displayed either an immediate or delayed increase in representation until reaching an apparent steady state between 72 and $120 \mathrm{hpf}$ with a 7 -fold extent of RNA variation (zGlyR $\alpha 2$ vs. zGlyRßb).

In parallel, we also performed whole-mount in situ hybridizations in an attempt to determine which tissues expressed zGlyR subunits. To this end we utilized 48 and $120 \mathrm{hpf}$ larvae as all zGlyR subunits were expressed at these time points (Figure $2 \mathbf{B}$ ). We found that $z G l y R \alpha 4$ a transcripts were present in eye, consistent with a previous report (Hensley et al., 

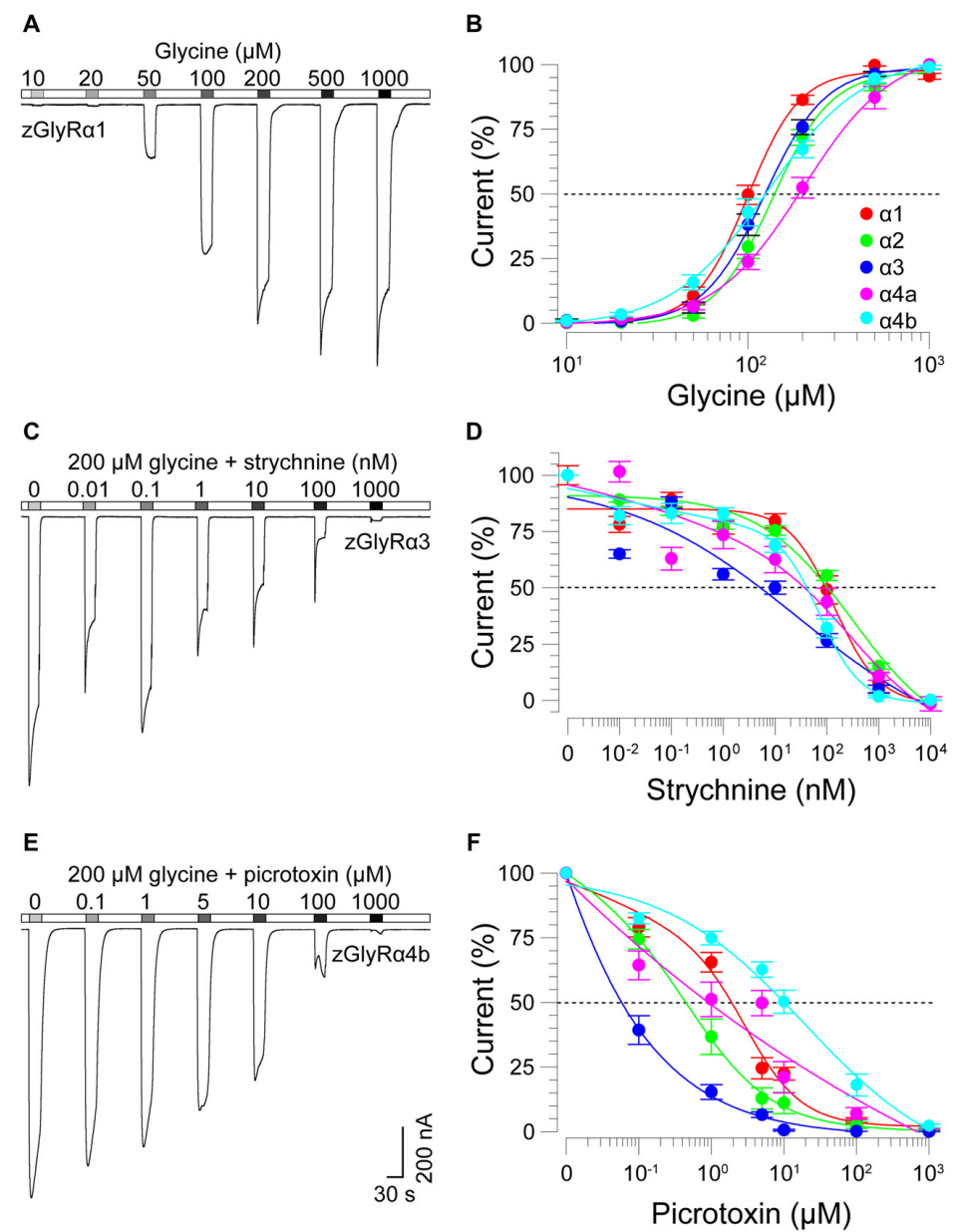

FIGURE 4 | zGlyR $\alpha$ subunits form functional homomeric receptors that are activated by glycine, and inhibited by strychnine and picrotoxin. (A) Two-electrode voltage-clamp recording from an oocyte injected with five femtomoles of zGlyR $\alpha 1$ cRNA exposed to serial application of glycine of increasing amount. Ten oocytes were used for each assay. (B) Cumulative dose-response relationship of glycine-evoked currents. The amplitude of each glycine-evoked response was normalized to the maximally-evoked current for each oocyte $(n=10)$. Values here and elsewhere represent the average \pm SEM. Dashed line denotes $E_{50}$. (C,E) Recordings from oocytes exposed to glycine and increasing amounts of strychnine or picrotoxin, respectively $(n=10)$. (D,F) Cumulative dose-response relationships of strychnine and picrotoxin-blocked currents $(n=10)$. Extent of blockade was normalized to the current amplitude evoked by $200 \mu \mathrm{M}$ glycine in each oocyte. Dashed lines denote $\mathrm{IC}_{50 \mathrm{~s}}$.

2011). Whereas all subunits were expressed in the hindbrain of $48 \mathrm{hpf}$ larvae (Figure 3), transcripts for zGlyR $\alpha 1, \mathrm{zGlyR} \alpha 2$, zGlyR $\alpha 4 a, z G l y R \alpha 4 b, z G l y R ß a$ and zGlyRßb were detected in the spinal cord. Likewise, at $120 \mathrm{hpf}$ all transcripts were again detected in the hindbrain (Supplementary Figure S1), while $z G l y R \alpha 1$ and $z G l y R \alpha 3$ were detected in the spinal cord. These findings were consistent with the observed expression pattern of mammalian GlyRs (Zarbin et al., 1981; Probst et al., 1986).

\section{zGlyR $\alpha$ Subunits Form Homomeric and Heteromeric Receptors With zGlyRß Subunits}

To determine the functionality of zGlyR subunits, we first made two-electrode voltage-clamp recordings from Xenopus oocytes injected with cRNA encoding a single subunit. This approach revealed that each $\mathrm{zGlyR} \alpha$ subunit, including the alternate variant of zGlyR $\alpha 4 a$ (Hirata et al., 2013), formed functional homomeric receptors that were activated by micromolar amounts of glycine (Figures 4A,B), and inhibited by nanomolar amounts of strychnine and micromolar amounts of picrotoxin (Figures 4C-F; Table 2). In contrast, both zGlyRß subunits failed to yield currents above background (not shown), a finding in agreement with the absence of necessary residues within assembly domain one of zGlyRß subunits (Griffon et al., 1999).

As $B$ subunits from other organisms complex with $\alpha$ subunits to form heteromeric receptors (Pfeiffer et al., 1982; Grenningloh et al., 1990; Pribilla et al., 1992), we next recorded from oocytes co-expressing each zGlyR $\alpha$ subunit and either zGlyRßa or zGlyRßb. We found that the co-injection of zGlyRß cRNAs consistently led to a rightward shift in the $\mathrm{EC}_{50}$ for glycine when compared to oocytes expressing each zGlyR $\alpha$ subunit alone 
TABLE 2 | Summary of homomeric and heteromeric zGlyR properties.

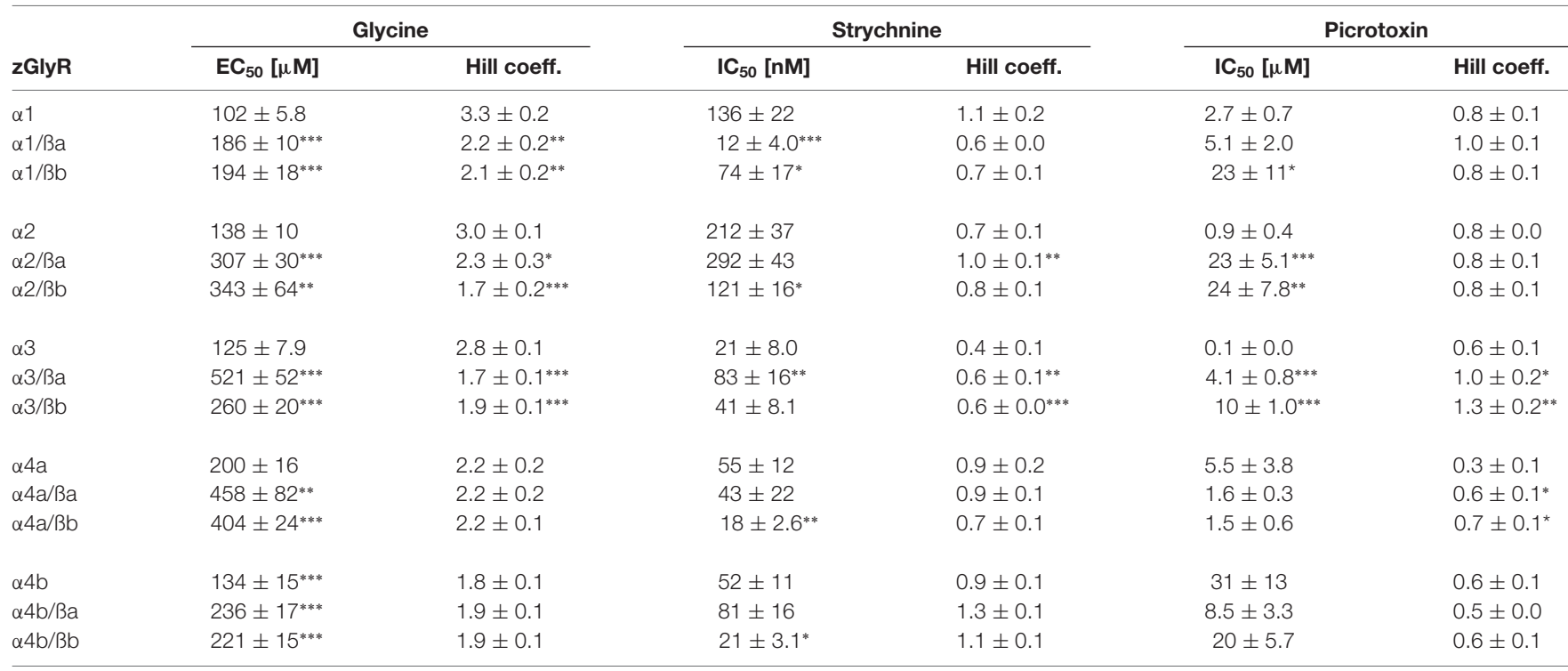

Values represent the average \pm SEM from 10 oocytes each. Where indicated ${ }^{*} p<0.05,{ }^{* *} p<0.01$, and ${ }^{* * *} p<0.001$ reflect difference between heteromeric and homomeric receptors.

(Figures 5A,B; Table 2); a result consistent with the formation of heteromeric GlyRs in oocytes (Kuhse et al., 1993; Langosch et al., 1994; Grudzinska et al., 2005). In contrast, the co-expression of zGlyRß subunits with $\mathrm{zGlyR} \alpha$ subunits either had no effect on strychnine's half-maximal inhibitory amount $\left(\mathrm{IC}_{50}\right)$, or yielded mixed results. For example, both zGlyRß subunits reduced strychnine's $\mathrm{IC}_{50}$ when co-expressed with $\mathrm{zGlyR} \alpha 1$, while zGlyRßb reduced the $\mathrm{IC}_{50}$ for $z G l y R \alpha 2$, zGlyR $\alpha 4 \mathrm{a}$ and zGlyR $\alpha 4 b$ (Figures 5C,D; Table 2). By comparison, zGlyRßa was found to increase strychnine's IC $_{50}$ when co-expressed with zGlyR $\alpha 3$.

Lastly, we explored the sensitivity of heteromeric zGlyRs to the pore-blocking antagonist picrotoxin. We found that oocytes expressing heteromeric zGlyR $\alpha 1 / ß a$ receptors were as sensitive to picrotoxin as those expressing homomeric zGlyR $\alpha 1$ receptors (Figures 5E,F). Likewise, heteromeric receptors comprised of zGlyR $\alpha 4$ a or zGlyR $\alpha 4 b$ and either zGlyRß paralog were also indistinguishable from their homomeric zGlyR $\alpha$ counterparts (Table 2 ). In contrast, zGlyRßb rendered heteromeric zGlyR $\alpha 1 / \beta$ b receptors $\sim 8.5$ fold more resistant to picrotoxin, while heteromeric receptors comprised of zGlyRßa or zGlyRßb and either zGlyR $\alpha 2$ or zGlyR $\alpha 3$ were between 25 and 100 fold more resistant. While the basis for the varying effects of zGlyRß paralogs on a receptor's sensitivity to strychnine and picrotoxin is currently unclear, these results are nonetheless consistent with the formation of functional heteromeric zGlyRs.

\section{Residues Within Transmembrane Domains Two and Three of Zebrafish and Human GlyRß Subunits Contribute to Picrotoxin Resistance}

To examine the molecular basis of picrotoxin sensitivity in heteromeric zGlyRs, we chose to focus on $z G l y R \alpha 1 / \beta$ receptors as previous research had identified essential residues within M2 of the human $ß$ subunit (hGlyRß) essential for picrotoxin resistance in heteromeric hGlyR $\alpha 1 / \beta$ receptors (Pribilla et al., 1992; Shan et al., 2001). A sequence comparison between the M2s of zGlyRß subunits and hGlyRß uncovered that zGlyRß subunits differ from hGlyRß at the 6', 12', and 17' position (Figure 1C). Given that the conversion of the 6' position in hGlyRß from phenylalanine to threonine abolishes $B$-subunit conferred picrotoxin resistance in heteromeric receptors, and that conversion of the same position in hGlyR $\alpha 1$ from glycine to phenylalanine confers picrotoxin resistance to homomeric receptors (Shan et al., 2001), we first substituted the leucines at the 6' position in both zGlyRß paralogs with phenylalanines (zGlyRßa ${ }^{\mathrm{L} 280 \mathrm{~F}}, \mathrm{zGlyR}^{\mathrm{L}} \mathrm{b}^{\mathrm{L} 281 \mathrm{~F}}$ ). We found that the resistance of $\mathrm{zGlyR} \alpha 1 / \mathrm{Ba}^{L 280 F}$ and zGlyR $\alpha 1 / \beta b^{L 281 F}$ receptors to picrotoxin was indistinguishable from wild-type $z G l y R \alpha 1 / \beta$ receptors (Table 3 ), indicating that the presence of an aromatic phenylalanine at the 6' position alone was insufficient to significantly increase picrotoxin resistance. We therefore, next made individual and combined substitutions of all divergent residues within M2, which revealed that conversion of all three residues (TM: triple mutant) induced an $\sim 8$-fold increase in picrotoxin resistance in heteromeric $\mathrm{zGlyR} \alpha 1 / \mathrm{Ba}^{\mathrm{TM}}$ receptors (Figure 6A). However, conversion of the same residues in heteromeric zGlyR $\alpha 1 / \beta^{\mathrm{TM}}$ receptors increased picrotoxin resistance by $\sim 42$ fold (Figure 6B); a finding which indicates that previously unidentified residues outside of M2 also contribute to picrotoxin resistance.

A further comparison of nearby residues revealed that zGlyRßa differs from zGlyRßb and hGlyRß subunits at the $7^{\prime}$ and $12^{\prime}$ positions within transmembrane domain 3 (M3; Figure 6C). Subsequent conversion of methionine to leucine at the $7^{\prime}$ position rendered $\mathrm{zGlyR} \alpha 1 / \mathrm{Ba}^{T M+M 309 L}$ 
A

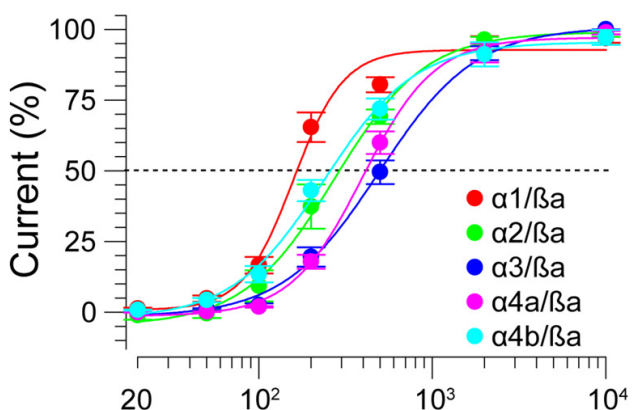

B

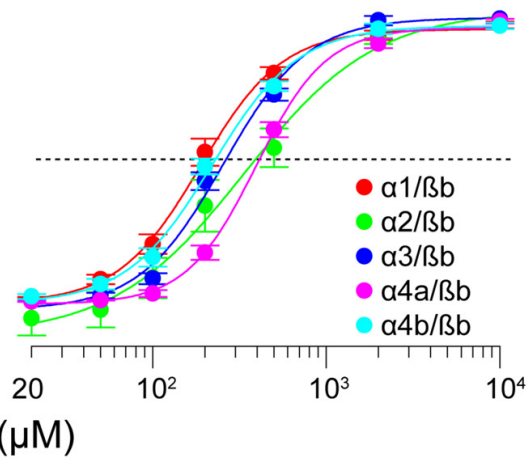

D

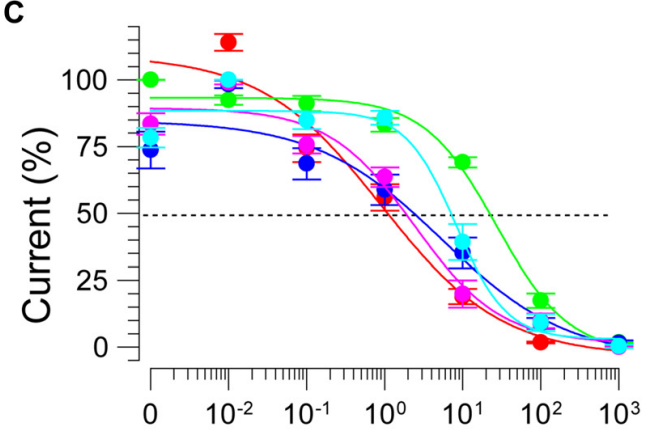

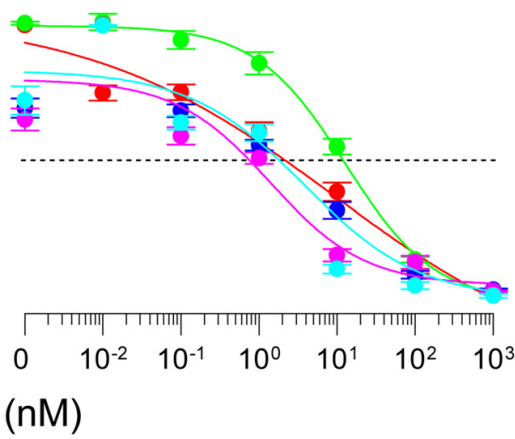

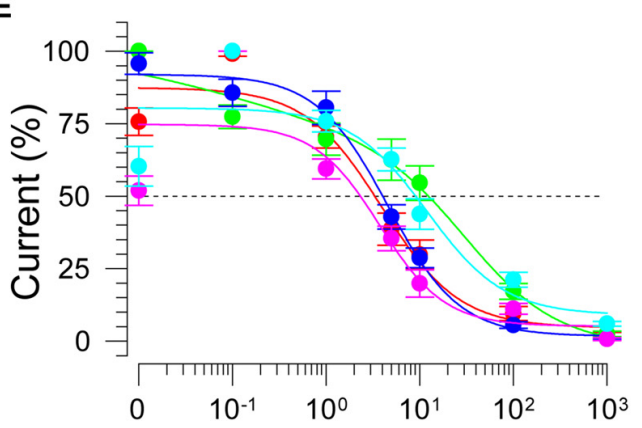

$\mathbf{F}$

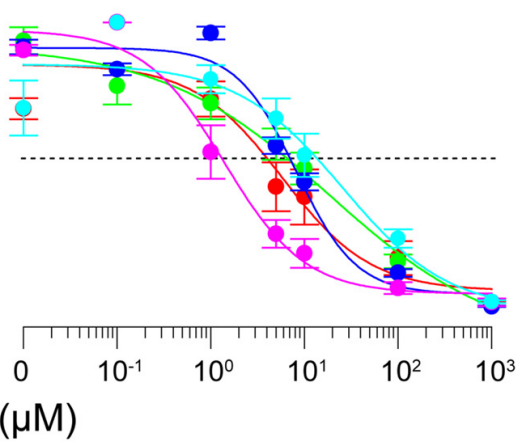

FIGURE 5 | zGlyRß subunits form functional heteromeric receptors with zGlyR $\alpha$ subunits. Cumulative dose-response relationships of glycine-evoked currents from oocytes co-injected with 2.5 femtomoles of zGlyR $\alpha$ cRNA and 2.5 femtomoles of either zGlyRßa (A) or zGlyRBb (B) cRNA. Ten oocytes were used for each assay. (C-F) Cumulative dose-response relationships of strychnine and picrotoxin-blocked currents from heteromeric zGlyRs. Ten oocytes were used for each assay.

receptors less resistant to picrotoxin (Table 3), while conversion of tyrosine at the $12^{\prime}$ position to phenylalanine yielded zGlyR $\alpha 1 / \mathrm{Ba}^{\mathrm{TM}+\mathrm{Y} 315 \mathrm{~F}}$ receptors that were $\sim 16$ fold more resistant (Figure 6A). To determine whether the analogous residue within hGlyRß also contributes to picrotoxin resistance we engineered a hGlyR $\beta^{F 317 Y}$ variant, which when co-expressed with hGlyR $\alpha 1$ yielded heteromeric receptors devoid of $B$ subunit-conferred picrotoxin resistance (Figure 6D), although we cannot completely exclude the possibility that hGlyRbF317Y subunit was not incorporated into functional GlyR heteromers. Taken together, residues within M2 and M3 of both zebrafish and human $ß$ subunits likely contribute to picrotoxin resistance.

\section{Mutations in zGlyRßb and zGlyR $\alpha 1$ Result in Hypomorphic Receptors}

Currently, seven alleles of the zebrafish mutant bandoneon exist (Granato et al., 1996; Hirata et al., 2005), which have been shown to arise from the following: three nonsense mutations that truncate $z G l y R ß b$ prior to the first transmembrane domain (not indicated), three missense mutations of unknown consequence (Figure 7A), and one adult-viable nonsense 
TABLE 3 | Summary of modified B subunits effects on picrotoxin and glycine sensitivity.

\begin{tabular}{|c|c|c|c|c|c|}
\hline zGlyR & B subunit & $\mathrm{IC}_{50}[\mu \mathrm{M}]$ & Hill coeff. & $\mathrm{EC}_{50}[\mu \mathrm{M}]$ & Hill coeff. \\
\hline$\alpha 1$ & none & $2.4 \pm 0.8$ & $0.9 \pm 0.1$ & $108 \pm 8.8$ & $3.2 \pm 0.1$ \\
\hline \multirow[t]{8}{*}{$\alpha 1 / \mathrm{Ba}$} & wild type & $5.0 \pm 1.3$ & $1.1 \pm 0.1$ & $191 \pm 11$ & $2.1 \pm 0.2$ \\
\hline & L280F & $6.3 \pm 1.8$ & $0.4 \pm 0.1^{* * *}$ & & \\
\hline & S291T & $2.3 \pm 0.4^{*}$ & $0.7 \pm 0.1^{* *}$ & & \\
\hline & L280F+S286A & $4.6 \pm 1.8$ & $0.9 \pm 0.2$ & & \\
\hline & L280F+S291T & $8.7 \pm 2.5$ & $0.6 \pm 0.1^{* *}$ & & \\
\hline & L280F+S286A+S291T+M309L & $10 \pm 2.0$ & $0.4 \pm 0.1^{* * *}$ & & \\
\hline & L280F+S286A+S291T+Y315F & $44 \pm 6.7^{* * *}$ & $0.4 \pm 0.1^{* * *}$ & $220 \pm 17$ & $2.7 \pm 0.3$ \\
\hline & L280F+S286A+S291T+M309L+Y315F & $26 \pm 5.9^{* *}$ & $0.4 \pm 0.2^{* *}$ & & \\
\hline \multirow[t]{4}{*}{$\alpha 1 / \beta b$} & wild type & $20 \pm 16$ & $0.7 \pm 0.2$ & $212 \pm 22$ & $2.2 \pm 0.2$ \\
\hline & L281F & $16 \pm 6.2$ & $0.5 \pm 0.2$ & & \\
\hline & S287A & $4.0 \pm 0.7$ & $0.8 \pm 0.1$ & & \\
\hline & L281F+S287A+S292T+F316Y & $40 \pm 6.8$ & $0.3 \pm 0.0^{*}$ & $153 \pm 18$ & $3.5 \pm 0.6$ \\
\hline \multicolumn{6}{|c|}{ s } \\
\hline$\alpha 1$ & none & $1.6 \pm 0.3$ & $0.6 \pm 0.0$ & $178 \pm 9.2$ & $3.2 \pm 0.1$ \\
\hline \multirow[t]{2}{*}{$\alpha 1 / \beta$} & wild type & $31 \pm 5.0$ & $0.1 \pm 0.0$ & $218 \pm 11$ & $2.2 \pm 0.2$ \\
\hline & F317Y & $1.9 \pm 0.1^{* * *}$ & $0.6 \pm 0.1^{* * *}$ & $167 \pm 18^{*}$ & $2.4 \pm 0.3$ \\
\hline
\end{tabular}

Values represent the average \pm SEM from 10 oocytes each. ${ }^{*} p<0.05,{ }^{* *} p<0.01$, and ${ }^{* * *} p<0.001$ reflect difference between wild type and modified heteromeric receptors.

mutation that truncates $\mathrm{zGlyR} ß \mathrm{~b}$ in the B-loop prior to the gephyrin-binding motif and the fourth transmembrane domain (Hirata et al., 2005; Ganser et al., 2013). In an attempt to elucidate the functional consequence of the three lethal missense mutations, and lone viable nonsense mutation, we co-expressed zGlyRßb subunits bearing each mutation with zGlyR $\alpha 1$ in oocytes. To facilitate the formation of heteromeric receptors we co-injected oocytes with zGlyR $\alpha 1$ and zGlyRßb cRNAs at a ratio of 1:4. Assuming an equal probability of subunit selection during receptor assembly, a 1:4 ratio predicts that 99.9\% of the GlyRs formed will be heteromeric receptors (see "Materials and Method" section). We found that the four mutations in zGlyRßb result in functional heteromeric receptors (Figure 7B). A closer inspection revealed that three of the four mutated receptors exhibited elevated $\mathrm{EC}_{50}$ for glycine, despite displaying similar current amplitudes in response to $10 \mathrm{mM}$ glycine. The fourth mutated receptor, zGlyR $\alpha 1 / \mathrm{Bb}^{K 343 X}$ was indistinguishable from wild-type heteromeric receptors, indicating that the mutant phenotype is not apparently caused by a defect in ligand binding or $\mathrm{Cl}^{-}$ conductance.

In parallel to the use of bandoneon mutants, two additional studies have sought to investigate the contribution of glycinergic signaling in zebrafish through the use of mutated zGlyR $\alpha$ subunits (Ganser et al., 2013; Leacock et al., 2018). In brief, a dominant form of hyperekplexia in humans is caused by mutations in hGlyR $\alpha 1$ that convert the arginine immediately following M2 to either leucine or glutamine (Shiang et al., 1993). Subsequent investigations revealed that the hGlyR $\alpha 1^{R 271 Q}$ mutation increases $\mathrm{EC}_{50}$ for glycine and results in severely diminished glycine-evoked currents owing to a $84 \%$ decrease in the single channel conductance of homomeric hGlyR $\alpha 1^{R 271 Q}$ receptors, and between a $\sim 150-180$-fold reduction in the sensitivity of homomeric and heteromeric receptors to glycine, respectively (Langosch et al., 1994). As this arginine residue is conserved among GlyR $\alpha$ subunits from different species (Hirata et al., 2009), substitution of the analogous residue in zGlyR $\alpha$ subunits is thought to exert a similar effect. To examine this possibility, we made recordings from oocytes injected with cRNA encoding venus-tagged $\mathrm{zGlyR} \alpha 1$ bearing an arginine 271 to glutamine substitution (Figure 7A; zGlyR $\alpha 1^{R 271 Q}$ ). We found that the $z G l y R \alpha 1^{R 271 Q}$ mutation resulted in $96 \%$ reduction in glycine-evoked currents (Figure $7 \mathrm{C}$ ), and a $\sim 170$-fold shift in the glycine $\mathrm{EC}_{50}$. When co-expressed with wild-type venustagged zGlyR $\alpha 1$, we observed a 3.4-fold increase in the $\mathrm{EC}_{50}$ for glycine, and a $33 \%$ reduction in glycine-evoked currents. Finally, co-expression of $z G l y R \alpha 1^{R 271 Q}$ with $z G l y R ß b$ resulted in a $\sim 19$-fold increase in the $\mathrm{EC}_{50}$ for glycine, and a $56 \%$ reduction in glycine-evoked currents. Considering that a $15 \%$ increase in the $\mathrm{EC}_{50}$ for glycine is sufficient to impair motor activity (zGlyRß ${ }^{Y 79 D}$; Figure $\left.7 \mathrm{~B}\right)$ suggests that the ectopic expression of $z G l y R \alpha 1^{R 271 Q}$ is likely to be an effective tool for disrupting glycinergic transmission in vivo.

Finally, to determine whether $\mathrm{zGlyR} \alpha 1^{R 271 Q}$ subunits traffic to the membrane like their human counterpart (Langosch et al., 1993), we examined the subcellular localization of venus-tagged zGlyR $\alpha 1$ subunits in HEK293T cells. This approach revealed that $\mathrm{zGlyR} \alpha 1^{R 271 Q}$ subunits were distributed similar to wild-type zGlyR $\alpha 1$ subunits, and in a manner consistent with membrane localization (Figure 7D). 
A

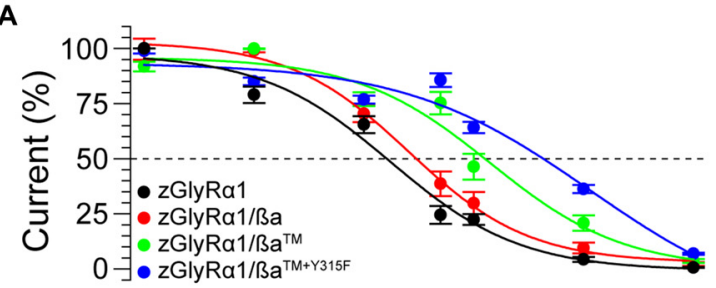

B

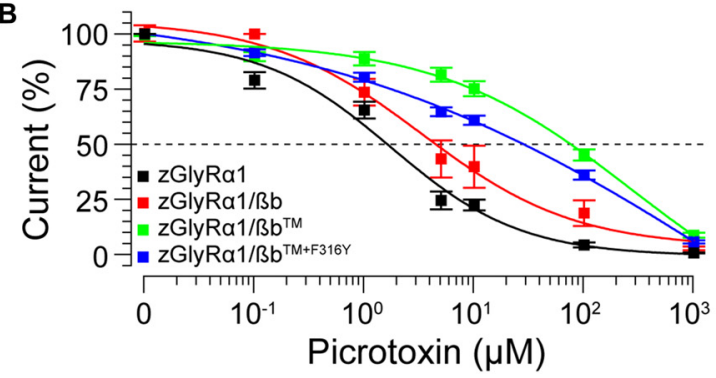

C
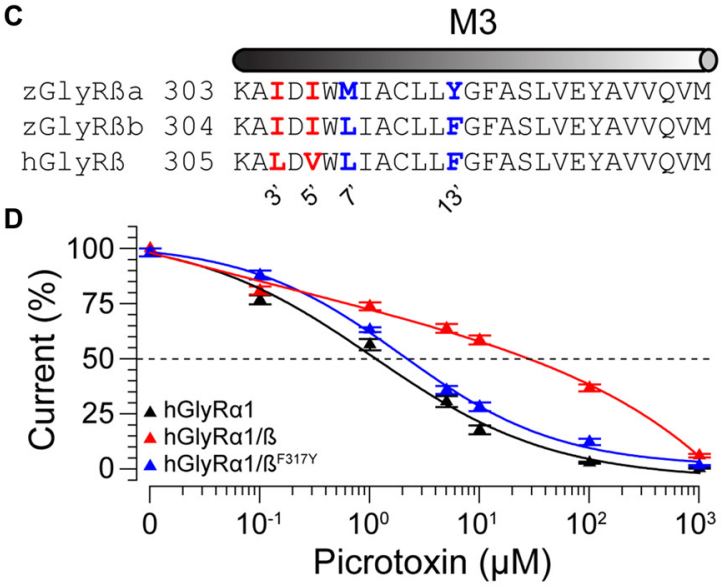

FIGURE 6 | Residues within M2 and M3 of zebrafish and human B subunits contribute to picrotoxin resistance. Cumulative effect of amino acid substitutions on picrotoxin-mediated blockade of zGlyRßa (A), zGlyRßb (B), and hGlyRB containing receptors (D). TM: triple mutation of residues in M2 of zGlyRB subunits; Ba: L280F+S286A+S291T, Bb: L281F+S287A+S292T. Ten oocytes were used for each assay. (C) Sequence alignments of M3 from hGlyRB and zGlyRß subunits. Red and blue letters indicate residues that are conserved between zGlyRß subunits, and zGlyRßb and hGlyRB, respectively.

\section{Heteromeric zGlyRs Assemble With a Subunit Stoichiometry of $3 \alpha: 2 \beta$}

Densiometric measurements of endogenous GlyRs purified from the spinal cords of rats using strychnine-based affinity chromatography uncovered $\alpha$ to $\beta$ subunit ratios most consistent with heteromeric receptors comprised of $3 \alpha: 2 \beta$ (Langosch et al., 1988). However, this technique might have led to an over estimation in the number of $\alpha$ subunits per heteromeric receptor given that a strychnine-based approach would also isolate pentameric GlyRs composed of only $\alpha$ subunits. Consistent with this possibility, subsequent studies employing a concatenated hGlyR $\alpha 1-\beta$ construct and metabolic labeling of monomeric subunits in Xenopus oocytes (Grudzinska et al., 2005), and atomic force microscopy measurements of antibody-labeled HEK293 cell extracts (Yang et al., 2012), have suggested an invariant heteromeric stoichiometry of $2 \alpha: 3 \beta$. To explore the subunit assembly of heteromeric zGlyRs we engineered a $\mathrm{zGlyR} \alpha 1-\beta \mathrm{b}$ concatenated construct and expressed it with either monomeric zGlyR $\alpha 1$ or zGlyRßb subunits (Figure 8A).

As a control, we first determined whether the concatenated construct alone was sufficient to generate functional channels. To this end we made recordings from oocytes injected with only cRNA encoding the zGlyR $\alpha 1-\beta b$ concatenated construct. We found that oocytes displayed minimal currents in response to $10 \mathrm{mM}$ glycine $(3.6 \pm 1.5 \mathrm{nA}$; Figure 8B), indicating a general absence of functional pseudo pentameric receptors featuring an outwardly facing sixth subunit. We therefore, next made recordings from oocytes co-injected with cRNA encoding the $\mathrm{zGlyR} \alpha 1-\beta b$ concatemer and either monomeric $z G l y R \alpha 1$ or monomeric zGlyRßb. We found that oocytes co-injected with concatenated $z G l y R \alpha 1-\beta b$ and monomeric zGlyRßb cRNAs again displayed minimal glycineevoked currents $(26.4 \pm 10.4 \mathrm{nA})$, indicating that $2 \alpha: 3 \beta$ heteromeric receptors are not readily made. In contrast, oocytes co-injected with concatenated zGlyR $\alpha 1-\beta b$ and monomeric zGlyR $\alpha 1$ cRNAs exhibited glycine-evoked responses reminiscent of currents obtained from oocytes co-injected with monomeric zGlyR $\alpha 1$ and monomeric zGlyRßb cRNAs (Figure 8C). Hence, heteromeric zGlyRs assemble with a stoichiometry of $3 \alpha: 2 \beta$.

\section{DISCUSSION}

Zebrafish have proven to be a useful model organism for studies related to glycinergic neurotransmission. For instance, mutagenesis screens have uncovered mutations in the zGlyRßb that result in bilateral contractions of the trunk and tail owing to a loss of reciprocal inhibition in the spinal cord (Hirata et al., 2005). The zebrafish bandoneon phenotype mirrors the human neurological disorder hyperekplexia which is likewise caused by mutations in hGlyR $\beta$ and hGlyR $\alpha 1$. This finding that simultaneously demonstrated the utility of using zebrafish to gain insight into human disorders, and highlighted the need for a better understanding of the zGlyR family. Here, we detail fundamental characteristics of all seven zGlyR subunits.

\section{The Potential Contribution of Maternally-Inherited RNA to Behavior and Neurogenesis}

The earliest behavior exhibited by zebrafish is spontaneous coiling of the trunk and tail. Spontaneous coiling begins at $17 \mathrm{hpf}$, peaks in frequency at $\sim 1 \mathrm{~Hz}$ at $19 \mathrm{hpf}$, and then dissipates incrementally over the next $7 \mathrm{~h}$ (Saint-Amant and Drapeau, 1998). Recordings made from spinal motor neurons between $19 \mathrm{hpf}$ and $20 \mathrm{hpf}$ have uncovered two types of electrical inputs to motor neurons that are thought to mediate coiling: gap-junction mediated periodic depolarizations that trigger bursts of action potentials, and activity-dependent 
A

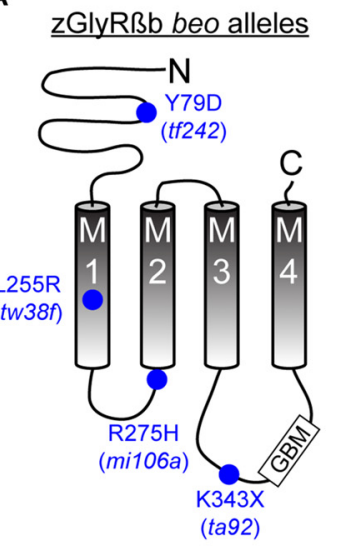

C

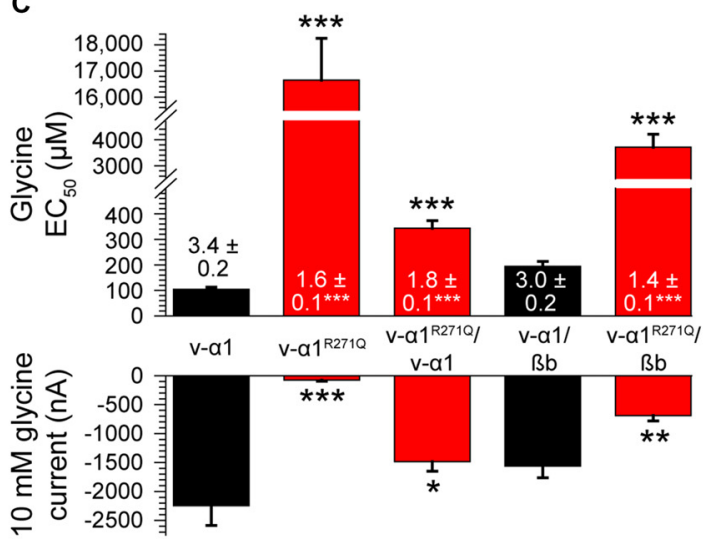

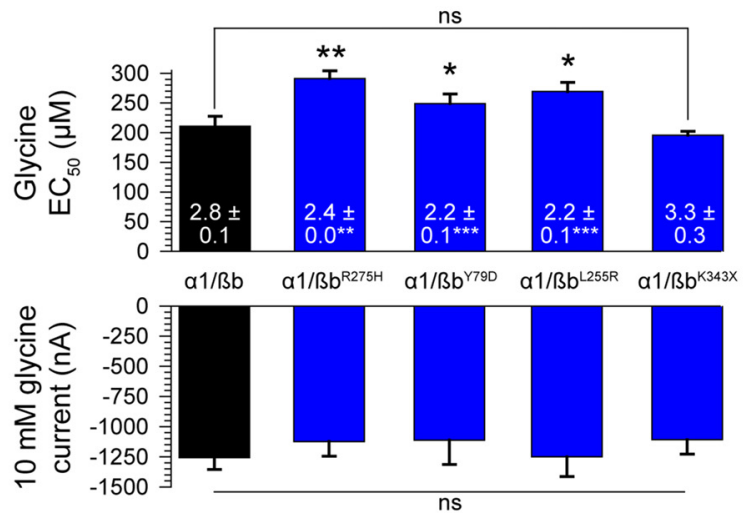

D

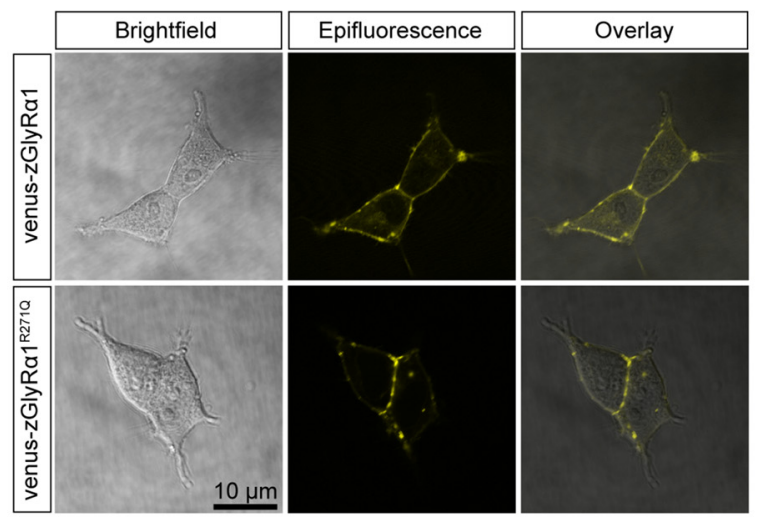

FIGURE 7 | Mutations in zGlyRßb and zGlyR $\alpha 1$ result in hypomorphic receptors. (A) Location of mutations in zGlyRßb that give rise to bandoneon (beo) mutants, and analogous position in hGlyR $\alpha 1$ of mutations that cause hyperekplexia. (GBM) Gephyrin-binding motif. (B) Cumulative effects of bandoneon mutations on heteromeric receptors' $\mathrm{EC}_{50}$ for glycine and average $10 \mathrm{mM}$ glycine-evoked currents. Of note, oocytes were injected with 1 femtomole of zGlyR $1 \mathrm{cRNA}$ and four femtomoles of zGlyRBb cRNAs. Ten oocytes were used for each assay. Numbers inside bar here and in (C) represent Hill coefficient, and ${ }^{*} p<0.05$, ${ }^{* *} p<0.01$, and ${ }^{* * *} p<0.001$. (C) Effect of dominant-negative R271Q mutation on both homomeric and heteromeric receptors' EC 50 for glycine and average 10 mM glycine-evoked currents. Each oocyte was injected with 2.5 femtomoles of venus-tagged zGlyR 1 cRNAs and 2.5 femtomoles of zGlyRßb cRNA. Ten oocytes were used for each assay. (D) Confocal images of HEK293T cells expressing venus-tagged zGlyR 1 subunits.

glycinergic synaptic bursts (Saint-Amant and Drapeau, 2000, 2001). Although glycine-mediated synaptic bursts are also depolarizing, owing to a $\mathrm{Cl}^{-}$equilibrium potential $\sim 20 \mathrm{mV}$ depolarized relative to the resting membrane potential (Reynolds et al., 2008), they fail to trigger action potentials. Instead, glycinergic input is thought to contribute to the coordination of alternating spontaneous coiling, and later swimming, by preventing the bilateral activation of motor neurons through a commissural inhibitory shunting effect. Consistent with this role, bandoneon mutants exhibit bilateral contractions (Granato et al., 1996), however, bilateral contractions are not readily apparent until $\geq 24 \mathrm{hpf}$ (Hirata et al., 2005). This finding suggests that activity-dependent glycinergic input between $20-23 \mathrm{hpf}$ is either dispensable for the coordination of coiling, or that a transient compensatory mechanism exists. In support of a transient compensatory mechanism was the detection of maternally inherited transcripts encoding zGlyR $\alpha 1, z G l y R \alpha 4 b$ and both zGlyRß paralogs (Figure 2B).
If maternally inherited zGlyRß mRNA initially compensates for the loss of embryonic zGlyRßb in bandoneon mutants, then translation-blocking morpholinos targeting the zGlyRß paralogs would be expected to exacerbate the onset of bilateral contractions.

In addition to a classical role in neurotransmission, glycinergic signaling in zebrafish has also been implicated in the neurogenesis of spinal interneurons. In brief, blockade of glycinemediated membrane depolarization by strychnine (McDearmid et al., 2006; Côté and Drapeau, 2012), or by rendering GlyR activation hyperpolarizing by reversing the $\mathrm{Cl}^{-}$gradient through the ectopic expression of the $\mathrm{Cl}^{-}$transporter KCC2 (Reynolds et al., 2008), result in reduced neurogenesis of spinal interneurons. Additional efforts revealed that GlyR-dependent membrane depolarizations are necessary for the activation of voltage-gated L-type calcium channels, which in turn is essential for neurogenesis (Brustein et al., 2013). Thus, the inheritance of maternal RNA encoding zGlyR subunits might be essential for 


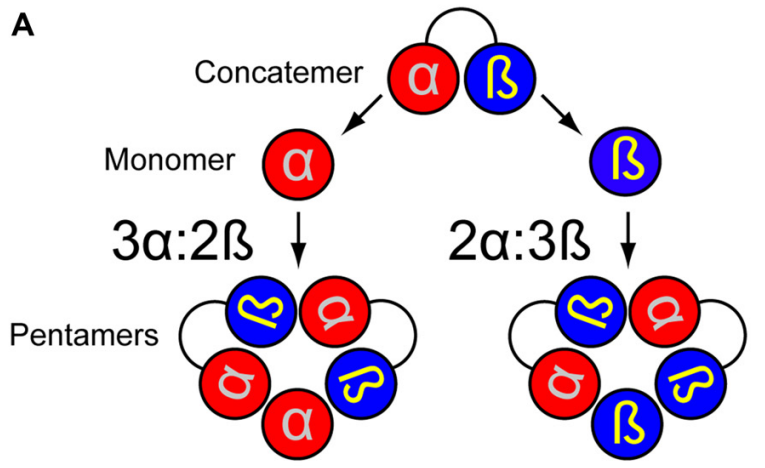

B
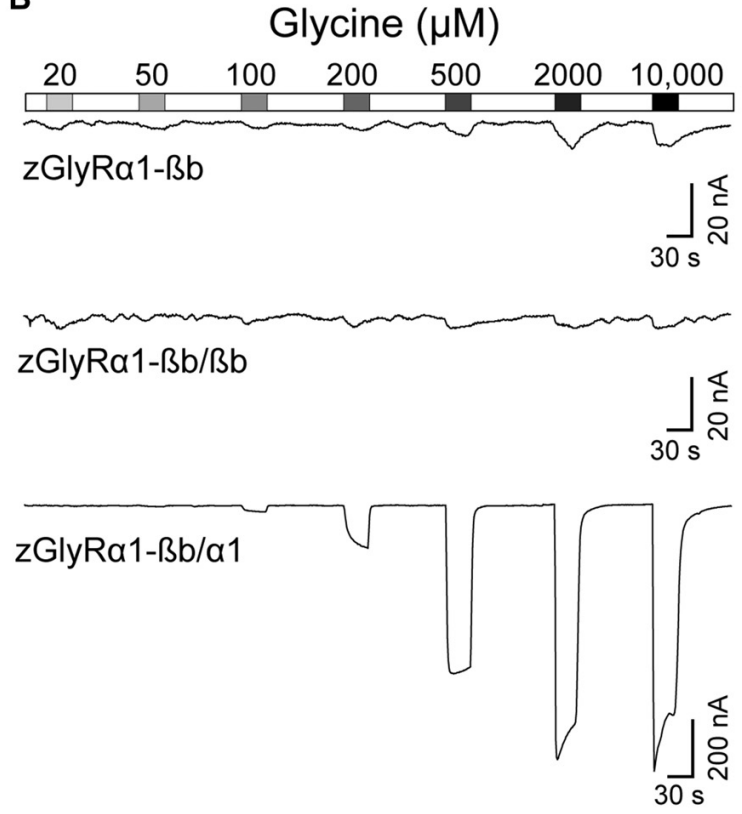

C

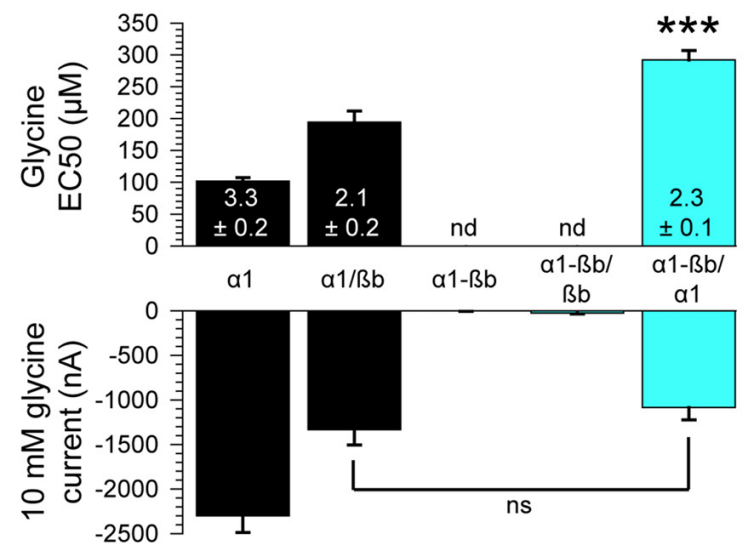

FIGURE 8 | Heteromeric zGlyRs assemble with a stoichiometry of $3 \alpha: 2 \beta$. (A) Schematic detailing the assembly of pentameric zGlyRs from concatemers and either monomeric zGlyR $\alpha 1$ or zGlyRßb. (B) Two-electrode voltage clamp recordings from oocytes injected with cRNA encoding concatemers alone, or with monomeric zGlyR $\alpha 1$ or zGlyRßb cRNA. Note that the current scale bars on the top and middle traces differ from the bottom trace. (C) Average glycine $\mathrm{EC}_{50}$ and responses to $10 \mathrm{mM}$ glycine $(n=10) .{ }^{* * *} p<0.001$. an early period GlyR-dependent neurogenesis, as well as in the shaping of an early behavior through synaptic transmission.

\section{Functional Aspects of zGlyR Subunits and Receptors}

Although the initially recovered signal peptide sequence of zGlyR $\alpha 4 a$ was found to be incapable of producing functional receptors (Imboden et al., 2001a), here, we report that an alternate variant of zGlyR $\alpha 4 \mathrm{a}$ containing a different signal peptide sequence capable of compensating for the loss of zGlyR expression in larvae (Hirata et al., 2013), yielded functional homomeric receptors (Figure 4F) with properties similar to mouse GlyR $\alpha 4$ (Harvey et al., 2000). Therefore, all seven zGlyR subunits are capable of contributing to glycinergic neurotransmission in zebrafish.

We also noted a difference in zGlyR $\alpha 4 a$ when co-expressed with zGlyRßa vs. zGlyRßb. In both instances, co-expression of zGlyR $\alpha 4 a$ with either zGlyRß paralog resulted in heteromeric receptors with a decreased sensitivity to glycine relative to homomeric receptors (Table 2). However, whereas zGlyRßa resulted in heteromeric receptors with a homomeric-like sensitivity to the competitive antagonist strychnine, heteromeric receptors comprised of zGlyRa4a and zGlyRßb exhibited increased sensitivity to strychnine. This phenomenon was not exclusive to zGlyR $\alpha 4 a$ as both $z G l y R \alpha 4 b$ and $z G l y R \alpha 2$ exhibited a similar pattern of decreased sensitivity to glycine when co-expressed with either zGlyRß paralog, but increased sensitivity to strychnine when co-expressed with zGlyRßb. Curiously this pattern was reversed with $z G l y R \alpha 1$, with heteromeric receptors comprised of zGlyRßa exhibiting increased sensitivity to strychnine relative to $z G l y R \alpha 1 / \beta b$ receptors. As no discernable pattern exist, these findings likely represent the differential interactions of variant amino acids between zGlyRßa and zGlyRßb that lie within or near the binding site, coupled with sequence variations between $z G l y R \alpha$ subunits.

It is worth noting that a similar effect of lowered glycine sensitivity in heteromeric receptors relative to homomeric receptors has been observed for hGlyR $\alpha 1$ and hGlyRß when co-expressed in Xenopus oocytes (Kuhse et al., 1993; Langosch et al., 1994; Grudzinska et al., 2005). In contrast, recordings from HEK cells co-expressing hGlyRß and either hGlyR $\alpha 1$, $\alpha 2$ or $\alpha 3$ have reported the formation heteromeric receptors with glycine sensitivities similar to homomeric receptors (Pribilla et al., 1992; Shan et al., 2001). Therefore, some of the differences between the receptors detailed here might represent phenomenon arising from the heterologous expression of GlyRs in oocytes.

\section{Stoichiometry of Heteromeric zGlyRs}

Experimental evidence concerning the subunit stoichiometry of heteromeric GlyRs is plentiful, but unfortunately conflicting. For instance, the first report assessing subunit stoichiometry using biochemical techniques suggested a stoichiometry most consistent with $3 \alpha: 2 \beta$ (Langosch et al., 1988). In support of this stoichiometry, subsequent investigations employing substitutions of presumptive pore-lining residues found that 
alterations in GlyR $\alpha$ subunits more drastically influenced heteromeric receptor characteristics than analogous alterations in GlyRß subunits (Burzomato et al., 2003). Furthermore, results obtained using $\alpha / \beta$ chimeras (Kuhse et al., 1993), and single-molecule imaging coupled with step-wise photobleaching (Durisic et al., 2012), lent further support to an invariant stoichiometry of $3 \alpha: 2 \beta$. However, parallel experiments employing atomic force microscopy measurements (Yang et al., 2012), a concatenated hGlyR $\alpha 1-\beta$ construct and radiometricbased metabolic labeling of monomeric subunits (Grudzinska et al., 2005), have instead suggested an invariant stoichiometry of $2 \alpha: 3 ß$. While our results favor a subunit stoichiometry of $3 \alpha: 2 ß$ (Figure 8), we failed to explore other combinations (i.e., $4 \alpha: 1 \beta$ and $1 \alpha: 4 \beta$ ), and therefore whether the stoichiometry of heteromeric zGlyRs is also invariant is currently unresolved. However, we can report that an apparent propensity for the formation of heteromeric receptors over homomeric receptors exists given that oocytes co-injected with $\alpha$ and $\beta$ cRNAs at ratios of 1:1 and 1:4 yielded glycine-evoked currents with identical EC50s and peak current amplitudes (Table 2 vs. Figure 7B). Future experiments will seek to determine whether zGlyRs also exhibit an invariant stoichiometry through the use zGlyR $\alpha 1-\alpha 1$ and zGlyRßb-ßb concatemers.

\section{Bandoneon Mutants}

Given the role of $ß$ subunits in the targeting of GlyRs to synapses, it is not surprising that mutations in zGlyRßb were recovered in mutagenesis screens. Of these mutants, the only viable allele arises from a premature truncation of $\mathrm{zGlyR} ß \mathrm{~b}$ prior to the gephyrin-binding motif and the fourth transmembrane domain. Despite lacking the last transmembrane domain, functional zGlyR $\alpha 1 / \mathrm{Bb}^{\mathrm{K} 343 \mathrm{X}}$ receptors indistinguishable from wild-type zGlyR $\alpha 1 / \beta$ b receptors were formed (Figure $7 \mathbf{B}$ ). While this finding might seem to account for the viability of homozygous $z G l y R ß b^{K 343 X}$ mutant fish, truncation of zGlyRßb prior to the gephyrin-binding motif predicts that $z G l y R \alpha / \beta b^{K 343 X}$ receptors would be absent from synapses in vivo. However, the viability of this allele suggests that glycinergic transmission is present, albeit likely reduced. If true, then another process independent of the zGlyRßb subunit's gephyrin-binding motif can facilitate the synaptic targeting of heteromeric GlyRs. Electrophysiological recordings from homozygous zGlyRßb ${ }^{K 343 X}$ mutant neurons, or the identification of synaptically localized zGlyRs via immunohistochemical labeling could provide evidence for the existence of an additional targeting mechanism.

In contrast to $z G l y R ß b^{K 343 X}$, the three lethal missense mutations were found to cause elevated $\mathrm{EC}_{50}$ s for glycine that ranged from $15 \%\left(\mathrm{zGlyR} ß \mathrm{~b}^{Y 79 D}\right)$ to $38 \%\left(\mathrm{zGlyR} \mathrm{b}^{R 275 H}\right)$. Of these, the decrease in the sensitivity of $z G l y R \alpha 1 / \beta b^{Y 79 D}$ receptors to glycine can be accounted for by the substitution of a negatively charged amino acid for a hydrophobic aromatic amino acid near the negative portion of the inter-subunit binding site for glycine (Figure 1C). By comparison the zGlyRßb ${ }^{L 255 R}$ and zGlyRßb ${ }^{R 275 H}$ mutations are located in M1 and at the cytoplasmic mouth of the M2 pore, and thereby distal to the ligand-binding domain for glycine (Figure 7A). Although the zGlyRßb $\mathrm{b}^{R 275 H}$ mutation might have been predicted to disrupt the conductance of $\mathrm{Cl}^{-}$ ions given that the conserved substitution of an arginine for a histidine introduces an aromatic ring at the mouth of the pore. However, both mutations yield normal amplitudes of glycineevoked currents (Figure 7B), suggesting that $\mathrm{Cl}^{-}$conductance through the receptors is largely undisturbed. Taken together, these findings suggest that both mutations affect glycine's ability to gate the channel in a manner similar to the hGlyR $\alpha 1^{G 254 P}$ substitution which results in a $\sim 6$ fold increase in the $\mathrm{EC}_{50}$ for glycine (Shan et al., 2001). Lastly, it is conceivable that additional effects on receptor function not assayed here exist, such as alterations in decay time constants, or that co-assembly with other zGlyR $\alpha$ subunits might result in additional defects.

\section{Future Perspectives}

The first seven bandoneon mutants were identified more than two decades ago (Granato et al., 1996). Since then, only one additional zGlyR subunit mutant has been isolated (Hirata et al., 2005), an eighth allele of bandoneon. Taken together, these mutagenesis efforts represent the screening of several thousand genomes, which although not reaching saturation, have nonetheless only uncovered mutations in zGlyRßb. It is somewhat surprising that mutations in other zGlyR subunits have not been recovered alongside the eight alleles of bandoneon given that all seven zGlyR subunits can contribute to functional receptors. This lack of mutations in other zGlyR subunits might represent that such mutations are lethal, or alternatively, might indicate a necessity of simultaneously disrupting several zGlyR subunits given that several zGlyR subunits exhibit overlapping expression patterns. Consistent with the latter possibility was the finding that a mutation in a RNA helicase essential for the production of mature mRNA encoding zGlyR $\alpha 1, z G l y R \alpha 3$ and zGlyR $\alpha 4 a$ (Hirata et al., 2013), and that the ectopic expression of dominant-negative GlyR subunits capable of disrupting zGlyRs comprised of any subunit combination, both cause motor impairment (Ganser et al., 2013; Leacock et al., 2018). Therefore, future attempts at gaining insight into the contribution of glycinergic neurotransmission in zebrafish through additional mutagenesis efforts, either forward or gene targeted using CRISPR/Cas9, might not be advisable. Instead, we propose the generation and use of a venus-tagged $z G l y R \alpha 1$ transgenic line bearing the R271Q mutation under the control of a UAS promoter. The venus-tagged $z G l y R \alpha 1^{R 271 Q}$ line would generate dominant-negative subunits capable of disrupting both homomeric and heteromeric zGlyRs. When combined with the appropriate Gal4 line, glycinergic transmission in any neuron of choice could be silenced.

Overall, the utility of using zebrafish for investigations into the contribution of glycinergic transmission to the formation and the functional of the neural circuits that underlie behavior has been established. Hopefully the work reported here will assist others in their pursuits related to glycinergic signaling.

\section{AUTHOR CONTRIBUTIONS}

SL and $\mathrm{HH}$ designed the research and wrote the manuscript. SL and DI performed the research and analyzed the data. 


\section{FUNDING}

The work reported here was funded in part by an Invitation Fellowship from the Japan Society for the Promotion of Science (to SL), a Grant-in-Aid for Scientific Research (B) and Scientific Research on Innovative Areas from the Ministry of Education, Culture, Sports, Science and Technology, the Naito Foundation, the Japan Epilepsy Research Foundation, and a Collaborative Research Grant from the National Institute of Genetics (to HH).

\section{ACKNOWLEDGMENTS}

We would like to thank Professor Robert Harvey (University of the Sunshine Coast, Australia) for providing human GlyR $\alpha 1$ and

\section{REFERENCES}

Betz, H., and Laube, B. (2006). Glycine receptors: recent insights into their structural organization and functional diversity. J. Neurochem. 97, 1600-1610. doi: 10.1111/j.1471-4159.2006.03908.x

Bormann, J., Rundstrom, N., Betz, H., and Langosch, D. (1993). Residues within transmembrane segment M2 determine chloride conductance of glycine receptor homo- and hetero-oligomers. EMBO J. 12, 3729-3737.

Brustein, E., Côté, S., Ghislain, J., and Drapeau, P. (2013). Spontaneous glycineinduced calcium transients in spinal cord progenitors promote neurogenesis. Dev. Neurobiol. 73, 168-175. doi: 10.1002/dneu.22050

Burzomato, V., Groot-Kormelink, P. J., Sivilotti, L. G., and Beato, M. (2003). Stoichiometry of recombinant heteromeric glycine receptors revealed by a pore-lining region point mutation. Receptors Channels 9, 353-361. doi: 10.3109/714041016

Chung, S. K., Bode, A., Cushion, T. D., Thomas, R. H., Hunt, C., Wood, S. E., et al. (2013). GLRB is the third major gene of effect in hyperekplexia. Hum. Mol. Genet. 22, 927-940. doi: 10.1093/hmg/dds498

Côté, S., and Drapeau, P. (2012). Regulation of spinal interneuron differentiation by the paracrine action of glycine. Dev. Neurobiol. 72, 208-214. doi: 10.1002/dneu.20972

David-Watine, B., Goblet, C., de Saint Jan, D., Fucile, S., Devignot, V., Bregestovski, P., et al. (1999). Cloning, expression and electrophysiological characterization of glycine receptor $\alpha$ subunit from zebrafish. Neuroscience 90 , 303-317. doi: 10.1016/s0306-4522(98)00430-8

Du, J., Lü, W., Wu, S., Cheng, Y., and Gouaux, E. (2015). Glycine receptor mechanism elucidated by electron cryo-microscopy. Nature 526, 224-229. doi: $10.1038 /$ nature 14853

Durisic, N., Godin, A. G., Wever, C. M., Heyes, C. D., Lakadamyali, M., and Dent, J. A. (2012). Stoichiometry of the human glycine receptor revealed by direct subunit counting. J. Neurosci. 32, 12915-12920. doi: 10.1523/JNEUROSCI.2050-12.2012

Dutertre, S., Becker, C. M., and Betz, H. (2012). Inhibitory glycine receptors: an update. J. Biol. Chem. 287, 40216-40223. doi: 10.1074/jbc.R112.408229

Ganser, L. R., Yan, Q., James, V. M., Kozol, R., Topf, M., Harvey, R. J., et al. (2013). Distinct phenotypes in zebrafish models of human startle disease. Neurobiol. Dis. 60, 139-151. doi: 10.1016/j.nbd.2013.09.002

Gielen, M., Thomas, P., and Smart, T. G. (2015). The desensitization gate of inhibitory Cys-loop receptors. Nat. Commun. 6:6829. doi: $10.1038 /$ ncomms7829

Granato, M., van Eeden, F. J., Schach, U., Trowe, T., Brand, M., Furutani-Seiki, M., et al. (1996). Genes controlling and mediating locomotion behavior of the zebrafish embryo and larva. Development 123, 399-413.

Grenningloh, G., Pribilla, I., Prior, P., Multhaup, G., Beyreuther, K., Taleb, O., et al. (1990). Cloning and expression of the $58 \mathrm{kd} \beta$ subunit of the inhibitory glycine receptor. Neuron 4, 963-970. doi: 10.1016/0896-6273(90)90149-a

Griffon, N., Büttner, C., Nicke, A., Kuhse, J., Schmalzing, G., and Betz, H. (1999). Molecular determinants of glycine receptor subunit assembly. EMBO J. 18, 4711-4721. doi: 10.1093/emboj/18.17.4711
GlyRß. We would also like to thank Yuta Katsuno and Yukie Arizono for experimental assistance, Yoshio and Miwako Machida, and other members of the Hirata lab for helpful comments, and assistance regarding the preparation of the manuscript and Steven Charles Low II for collecting data. We dedicate this research article to the talented scientist Sean Eric Low (11 March 1976-9 May 2018) killed in a tragic hit-and-run accident in Sagamihara, Japan.

\section{SUPPLEMENTARY MATERIAL}

The Supplementary Material for this article can be found online at: https://www.frontiersin.org/articles/10.3389/fnmol. 2018.00286/full\#supplementary-material

Grudzinska, J., Schemm, R., Haeger, S., Nicke, A., Schmalzing, G., Betz, H., et al. (2005). The $\beta$ subunit determines the ligand binding properties of synaptic glycine receptors. Neuron 45, 727-739. doi: 10.1016/j.neuron.2005. 01.028

Harvey, R. J., Schmieden, V., Von Holst, A., Laube, B., Rohrer, H., and Betz, H. (2000). Glycine receptors containing the $\alpha 4$ subunit in the embryonic sympathetic nervous system, spinal cord and male genital ridge. Eur. J. Neurosci. 12, 994-1001. doi: 10.1046/j.1460-9568.2000.00993.x

Hensley, M. R., Emran, F., Bonilla, S., Zhang, L., Zhong, W., Grosu, P., et al. (2011). Cellular expression of Smarca4 (Brg1)-regulated genes in zebrafish retinas. BMC Dev. Biol. 11:45. doi: 10.1186/1471-213x-11-45

Hirata, H., Carta, E., Yamanaka, I., Harvey, R. J., and Kuwada, J. Y. (2009). Defective glycinergic synaptic transmission in zebrafish motility mutants Front. Mol. Neurosci. 2:26. doi: 10.3389/neuro.02.026.2009

Hirata, H., Ogino, K., Yamada, K., Leacock, S., and Harvey, R. J. (2013). Defective escape behavior in DEAH-box RNA helicase mutants improved by restoring glycine receptor expression. J. Neurosci. 33, 14638-14644. doi: 10.1523/JNEUROSCI.1157-13.2013

Hirata, H., Saint-Amant, L., Downes, G. B., Cui, W. W., Zhou, W., Granato, M., et al. (2005). Zebrafish bandoneon mutants display behavioral defects due to a mutation in the glycine receptor $\beta$-subunit. Proc. Natl. Acad. Sci. U S A 102, 8345-8350. doi: 10.1073/pnas.0500862102

Huang, X., Chen, H., Michelsen, K., Schneider, S., and Shaffer, P. L. (2015). Crystal structure of human glycine receptor- $\alpha 3$ bound to antagonist strychnine. Nature 526, 277-280. doi: 10.1038/nature 14972

Imboden, M., De Saint Jan, D., Leulier, F., Korn, H., Goblet, C., and Bregestovski, P. (2001a). Isolation and characterization of an $\alpha 2$-type zebrafish glycine receptor subunit. Neuroscience 103, 799-810. doi: 10.1016/s03064522(00)00575-3

Imboden, M., Devignot, V., and Goblet, C. (2001b). Phylogenetic relationships and chromosomal location of five distinct glycine receptor subunit genes in the teleost Danio rerio. Dev. Genes Evol. 211, 415-422. doi: 10.1007/s004270100164

Imboden, M., Devignot, V., Korn, H., and Goblet, C. (2001c). Regional distribution of glycine receptor messenger RNA in the central nervous system of zebrafish. Neuroscience 103, 811-830. doi: 10.1016/s0306-4522(00)00576-5

James, V. M., Bode, A., Chung, S. K., Gill, J. L., Nielsen, M., Cowan, F. M., et al. (2013). Novel missense mutations in the glycine receptor $\beta$ subunit gene (GLRB) in startle disease. Neurobiol. Dis. 52, 137-149. doi: 10.1016/j.nbd.2012. 12.001

Kimmel, C. B., Ballard, W. W., Kimmel, S. R., Ullmann, B., and Schilling, T. F. (1995). Stages of embryonic development of the zebrafish. Dev. Dyn. 203, 253-310. doi: 10.1002/aja.1002030302

Kuhse, J., Laube, B., Magalei, D., and Betz, H. (1993). Assembly of the inhibitory glycine receptor: identification of amino acid sequence motifs governing subunit stoichiometry. Neuron 11, 1049-1056. doi: 10.1016/08966273(93)90218-g

Langosch, D., Herbold, A., Schmieden, V., Borman, J., and Kirsch, J. (1993). Importance of Arg-219 for correct biogenesis of $\alpha 1$ homooligomeric glycine receptors. FEBS Lett. 336, 540-544. doi: 10.1016/0014-5793(93)80872-r 
Langosch, D., Laube, B., Rundström, N., Schmieden, V., Bormann, J., and Betz, H. (1994). Decreased agonist affinity and chloride conductance of mutant glycine receptors associated with human hereditary hyperekplexia. EMBO J. 13, 4223-4228.

Leacock, S., Syed, P., James, V. M., Bode, A., Kawakami, K., Keramidas, A., et al. (2018). Structure/function studies of the $\alpha 4$ subunit reveal evolutionary loss of a GlyR subtype involved in startle and escape responses. Front. Mol. Neurosci. 11:23. doi: 10.3389/fnmol.2018.00023

Langosch, D., Thomas, L., and Betz, H. (1988). Conserved quaternary structure of ligand-gated ion channels: the postsynaptic glycine receptor is a pentamer. Proc. Natl. Acad. Sci. U S A 85, 7394-7398. doi: 10.1073/pnas.85. 19.7394

Low, S. E., Amburgey, K., Horstick, E., Linsley, J., Sprague, S. M., Cui, W. W., et al. (2011). TRPM7 is required within zebrafish sensory neurons for the activation of touch-evoked escape behaviors. J. Neurosci. 31, 11633-11644. doi: 10.1523/JNEUROSCI.4950-10.2011

Lynch, J. W. (2004). Molecular structure and function of the glycine receptor chloride channel. Physiol. Rev. 84, 1051-1095. doi: 10.1152/physrev.000 42.2003

Lynch, J. W. (2009). Native glycine receptor subtypes and their physiological roles. Neuropharmacology 56, 303-309. doi: 10.1016/j.neuropharm.2008. 07.034

McDearmid, J. R., Liao, M., and Drapeau, P. (2006). Glycine receptors regulate interneuron differentiation during spinal network development. Proc. Natl. Acad. Sci. U S A 103, 9679-9684. doi: 10.1073/pnas.0504871103

Meyer, G., Kirsch, J., Betz, H., and Langosch, D. (1995). Identification of a gephyrin binding motif on the glycine receptor $\beta$ subunit. Neuron $15,563-572$. doi: 10.1016/0896-6273(95)90145-0

Pfeiffer, F., Graham, D., and Betz, H. (1982). Purification by affinity chromatography of the glycine receptor of rat spinal cord. J. Biol. Chem. 257, 9389-9393.

Pribilla, I., Takagi, T., Langosch, D., Bormann, J., and Betz, H. (1992). The atypical M2 segment of the $\beta$ subunit confers picrotoxinin resistance to inhibitory glycine receptor channels. EMBO J. 11, 4305-4311.

Probst, A., Cortés, R., and Palacios, J. M. (1986). The distribution of glycine receptors in the human brain. A light microscopic autoradiographic study using $[3 \mathrm{H}]$ strychnine. Neuroscience 17, 11-35. doi: 10.1016/03064522(86)90222-8

Rees, M. I., Lewis, T. M., Kwok, J. B., Mortier, G. R., Govaert, P., Snell, R. G., et al. (2002). Hyperekplexia associated with compound heterozygote mutations in the $\beta$-subunit of the human inhibitory glycine receptor (GLRB). Hum. Mol. Genet. 11, 853-860. doi: 10.1093/hmg/11.7.853

Reynolds, A., Brustein, E., Liao, M., Mercado, A., Babilonia, E., Mount, D. B., et al. (2008). Neurogenic role of the depolarizing chloride gradient revealed by global overexpression of KCC2 from the onset of development. J. Neurosci. 28, 1588-1597. doi: 10.1523/JNEUROSCI.3791-07.2008

Saint-Amant, L., and Drapeau, P. (1998). Time course of the development of motor behaviors in the zebrafish embryo. J. Neurobiol. 37, 622-632. doi: 10.1002/(sici)1097-4695(199812)37:4<622::aid-neu10>3.0.co;2-s
Saint-Amant, L., and Drapeau, P. (2000). Motoneuron activity patterns related to the earliest behavior of the zebrafish embryo. J. Neurosci. 20, 3964-3972. doi: 10.1523/JNEUROSCI.20-11-03964.2000

Saint-Amant, L., and Drapeau, P. (2001). Synchronization of an embryonic network of identified spinal interneurons solely by electrical coupling. Neuron 31, 1035-1046. doi: 10.1016/s0896-6273(01)00416-0

Schindelin, J., Arganda-Carreras, I., Frise, E., Kaynig, V., Longair, M., Pietzsch, T., et al. (2012). Fiji: an open-source platform for biological-image analysis. Nat. Methods 9, 676-682. doi: 10.1038/nmeth.2019

Schmieden, V., Kuhse, J., and Betz, H. (1993). Mutation of glycine receptor subunit creates $\beta$-alanine receptor responsive to GABA. Science 262, 256-258. doi: $10.1126 /$ science. 8211147

Shan, Q., Haddrill, J. L., and Lynch, J. W. (2001). A single $\beta$ subunit M2 domain residue controls the picrotoxin sensitivity of $\alpha \beta$ heteromeric glycine receptor chloride channels. J. Neurochem. 76, 1109-1120. doi: 10.1046/j.1471-4159.2001. 00124.x

Shiang, R., Ryan, S. G., Zhu, Y. Z., Hahn, A. F., O'Connell, P., and Wasmuth, J. J. (1993). Mutations in the $\alpha 1$ subunit of the inhibitory glycine receptor cause the dominant neurologic disorder, hyperekplexia. Nat. Genet. 5, 351-358. doi: 10.1038/ng1293-351

Simon, J., Wakimoto, H., Fujita, N., Lalande, M., and Barnard, E. A. (2004). Analysis of the set of $\mathrm{GABA}_{\mathrm{A}}$ receptor genes in the human genome. J. Biol. Chem. 279, 41422-41435. doi: 10.1074/jbc.M401354200

Vandenberg, R. J., French, C. R., Barry, P. H., Shine, J., and Schofield, P. R. (1992). Antagonism of ligand-gated ion channel receptors: two domains of the glycine receptor $\alpha$ subunit form the strychnine-binding site. Proc. Natl. Acad. Sci. US A 89, 1765-1769. doi: 10.1073/pnas.89.5.1765

Wood, R. (1998). Trends in Genetics: Genetic Nomenclature Guide with Information on Websites. England: Elsevier Trends Journals.

Yang, Z., Taran, E., Webb, T. I., and Lynch, J. W. (2012). Stoichiometry and subunit arrangement of $\alpha 1 \beta$ glycine receptors as determined by atomic force microscopy. Biochemistry 51, 5229-5231. doi: 10.1021/bi300063m

Zarbin, M. A., Wamsley, J. K., and Kuhar, M. J. (1981). Glycine receptor: light microscopic autoradiographic localization with $[3 \mathrm{H}]$ strychnine. J. Neurosci. 1 , 532-547. doi: 10.1523/JNEUROSCI.01-05-00532.1981

Conflict of Interest Statement: The authors declare that the research was conducted in the absence of any commercial or financial relationships that could be construed as a potential conflict of interest.

The handling editor declares a past co-authorship with one of the authors that did not involve direct scientific collaboration, $\mathrm{HH}$, in 2018.

Copyright (c) 2018 Low, Ito and Hirata. This is an open-access article distributed under the terms of the Creative Commons Attribution License (CC BY). The use, distribution or reproduction in other forums is permitted, provided the original author(s) and the copyright owner(s) are credited and that the original publication in this journal is cited, in accordance with accepted academic practice. No use, distribution or reproduction is permitted which does not comply with these terms. 